\title{
Unconventional pairing and electronic dimerization instabilities in the doped Kitaev-Heisenberg model
}

\author{
Daniel D. Scherer, ${ }^{1}$ Michael M. Scherer, ${ }^{2}$ Giniyat Khaliullin, ${ }^{3}$ Carsten Honerkamp, ${ }^{4}$ and Bernd Rosenow ${ }^{1,5}$ \\ ${ }^{1}$ Institut für Theoretische Physik, Universität Leipzig, D-04103 Leipzig, Germany \\ ${ }^{2}$ Institut für Theoretische Physik, Universität Heidelberg, D-69120 Heidelberg, Germany \\ ${ }^{3}$ Max-Planck-Institut für Festkörperforschung, D-70569 Stuttgart, Germany \\ ${ }^{4}$ Institute for Theoretical Solid State Physics, RWTH Aachen University, D-52056 Aachen, \\ Germany and JARA - FIT Fundamentals of Future Information Technology, Germany \\ ${ }^{5}$ Physics Department, Harvard University, Cambridge, Massachusetts 02138, USA
}

\begin{abstract}
We study the quantum many-body instabilities of the $t-J_{\mathrm{K}}-J_{\mathrm{H}}$ Kitaev-Heisenberg Hamiltonian on the honeycomb lattice as a minimal model for a doped spin-orbit Mott insulator. This spin- $1 / 2$ model is believed to describe the magnetic properties of the layered transition-metal oxide $\mathrm{Na}_{2} \mathrm{IrO}_{3}$. We determine the ground-state of the system with finite charge-carrier density from the functional renormalization group (fRG) for correlated fermionic systems. To this end, we derive fRG flowequations adapted to the lack of full spin-rotational invariance in the fermionic interactions, here represented by the highly frustrated and anisotropic Kitaev exchange term. Additionally employing a set of Ward identities for the Kitaev-Heisenberg model, the numerical solution of the flow equations suggests a rich phase diagram emerging upon doping charge carriers into the ground-state manifold $\left(\mathbb{Z}_{2}\right.$ quantum spin liquids and magnetically ordered phases). We corroborate superconducting triplet $p$-wave instabilities driven by ferromagnetic exchange and various singlet pairing phases. For filling $\delta>1 / 4$, the $p$-wave pairing gives rise to a topological state with protected Majorana edge-modes. For antiferromagnetic Kitaev and ferromagnetic Heisenberg exchange we obtain bond-order instabilities at van Hove filling supported by nesting and density-of-states enhancement, yielding dimerization patterns of the electronic degrees of freedom on the honeycomb lattice. Further, our flow equations are applicable to a wider class of model Hamiltonians.
\end{abstract}

PACS numbers:

\section{INTRODUCTION}

The postulation of new topological states of matter and the quest for unraveling their properties has spurred a huge amount of research activity over the last decade, building on the groundbreaking insight that the appearance of the integer quantum Hall effect (IQHE) ${ }^{1 / 2}$ in twodimensional electron gases subject to quantizing perpendicular magnetic fields is intimately related to the topology of wavefunctions 3 . The prediction of the existence of a topological state generated by the presence of spinorbit coupling in $\mathrm{HgTe} / \mathrm{CdTe}$ quantum wells ${ }^{6 / 7}$ effectively opened out in the creation of the field of toplogical insulators and superconductors 8 12. While the IQHE still serves as a time-honored prototype to the field, many different models have been identified featuring the same or similar kinds of topological non-triviality in their wavefunctions 8 . 12 .

Another sub-field of condensed matter physics with strong connections to topology is the search for quantum spin liquids 13 - non-magnetic phases with typically exotic excitations and topological order ${ }^{14}[15$, a concept first introduced in the context of the fractional quantum Hall effect (FQHE) 16. On the theoretical side, the honeycomb Kitaev mode ${ }^{20}$ provided the first exactly solvable model with a quantum spin liquid ground-state in two spatial dimensions. Though a pure spin model, its excitations are Majorana fermions obeying non-Abelian exchange statistics 20 .
A route to explore Kitaev physics in a transition-metal oxide solid-state system, however, was suggested only recently. The naturally large spin-orbit and crystal-field energy scales in layered transition-metal oxides and the rather strong correlation effects in $5 d$ orbitals lead to highly anisotropic exchange interactions, entangling spin and orbital degrees of freedom. As a candidate compound, the layered honeycomb iridate $\mathrm{Na}_{2} \mathrm{IrO}_{3}$ was proposed $21 \mid 22$. It turned out to order magnetically below $T_{\mathrm{N}} \simeq 15 \mathrm{~K}$ in a so-called zigzag pattern different from the Néel state on the bipartite honeycomb lattice 2331 . Such a low ordering temperature was further taken as a sign of strongly frustrated exchange - a hallmark of Kitaev physics. Indeed, an effective (iso-)spin 1/2 model for the two states in the $j_{\text {eff }}=1 / 2$ part of the spinorbit split manifold of $t_{2 g}$ electrons was suggested to capture the magnetic properties of the Mott-insulating ground-state. It is simply given by Kitaev exchange and isotropic Heisenberg exchange for (iso-)spin $1 / 2$ degrees of freedom on nearest-neighbor sites in the honeycomb lattice. As the character of exchange interactions is varied among the possibilities of ferromagnetic and antiferromagnetic coupling, the model features a ground-state manifold with several interesting magnetic orderings with characteristic imprints on the spin-wave excitation spectrum. The $\mathbb{Z}_{2}$ quantum spin liquid ground state occurs for either ferromagnetic or antiferromagnetic Kitaev exchange, as long as the perturbation due to the Heisenberg coupling remains sufficiently small.

While the adequacy of the Kitaev-Heisenberg Hamil- 
tonian as a minimal model for the magnetic properties of the honeycomb iridate $\mathrm{Na}_{2} \mathrm{IrO}_{3}$ is still subject to debate ${ }^{32}$, the idea of studying the unconventional pairing states of the doped system has attracted additional attention from theory 33 . The resulting Hamiltonian can be understood as a paradigmatic model of a spin-orbit coupled, frustrated and doped Mott insulator. Besides singlet pairing phases, mean-field studies revealed $p$-wave triplet pairing phase ${ }^{33} \sqrt[35]{3}$, similar to the $B$-phase in ${ }^{3} \mathrm{He}$. Finally, when doping the system beyond quarter filling, the $p$-wave (mean-field) states are guaranteed $1011137 / 39$ to undergo a transition to a topological $p$-wave triplet

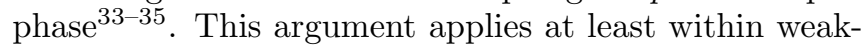
pairing theory. Almost in reminiscence of the Majorana excitations of the $\mathbb{Z}_{2}$ quantum spin liquid of the pure Kitaev model, the topological $p$-wave phase would posses Majorana states at vortex cores and Majorana edge modes propagating along the boundaries of the system. Amazingly, this line of investigation unveils the possible unification of the two different branches of topological insulator/superconductors and topologically ordered phases in the phase diagram of a paradigmatic single model Hamiltonian.

Regarding the possibility to experimentally realize doped states of the honeycomb iridate $\mathrm{Na}_{2} \mathrm{IrO}_{3}$, we would like to mention that electron doping of this material has been achieved recently by covering the sample with a potassium layer $\frac{40}{2}$. Further, doping of $\mathrm{Sr}_{2} \mathrm{IrO}_{4}$ iridium compounds became possible by the same technique and pseudogap physics as in cuprates was observed $\underline{41}$. Studying minimal models of doped spin-orbit Mott insulators therefore seems a worthwhile enterprise with possible connections to future experiments.

In this work, we investigate the phase diagram of the doped Kitaev-Heisenberg model beyond mean-field theory. This aim is accomplished within the flexible flow equation approach provided by the functional renormalization group $\underline{42}$ (fRG).

\section{MODEL, RESULTS AND OUTLINE}

In the following, we introduce the Hamiltonian of the doped Kitaev-Heisenberg model, see Subsect IIA. Our overall goal is to draw the ground-state phase diagram of the system, paying attention to different, competing ordering tendencies. We summarize and describe the gist of our results in Subsect. IIB demonstrating the richness of the phase diagram borne by doping of the KitaevHeisenberg system. We then provide an outline of this work in Subsect. IIC] Details are then provided in subsequent sections.

\section{A. The doped Kitaev-Heisenberg Model}

We study the Hamiltonian Eq. (1) on the honeycomb lattice as a minimal model for a doped spin-orbit Mott in-

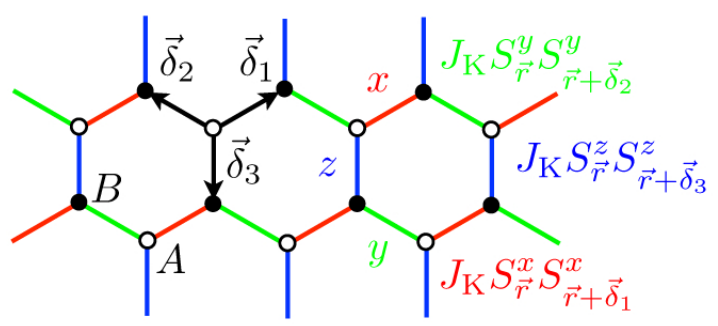

FIG. 1: Three plaquettes on the honeycomb lattice. White disks mark sites of the $A$-sublattice, while black disks those residing in the $B$-sublattice. Shown are also the three nearestneighbor vectors $\vec{\delta}_{i}, i=1,2,3$ pointing from a site in the $A$ sublattice to its nearest neighbors in the $B$-sublattice. The different colors of the bonds linking the sites of the honeycomb encode the bond-specific nearest-neighbor interaction of the Ising-like Kitaev exchange entering the Kitaev-Heisenberg Hamiltonian. Red bonds are called $x$-bonds and correspondingly, along an $x$-bond the $x$-components of neighboring spin operators are exchange-coupled. The conventions for $y$ - and $z$-bonds are analogous.

sulator. The Hamiltonian includes a kinetic part, which describes the hopping of the electrons, a Kitaev coupling $J_{\mathrm{K}}$, describing a bond-dependent Ising-like spin exchange, and a Heisenberg exchange, including a nearestneighbor density-density interaction of magnitude $J_{\mathrm{H}}$ that occurs upon doping. The Hamiltonian for the doped spin-orbit Mott insulator thus reads

$$
H=H_{\mathrm{kin}}+H_{\mathrm{K}}+H_{\mathrm{H}},
$$

where $^{1}$

$$
\begin{aligned}
H_{\text {kin }}= & -t_{0} \mathcal{P} \sum_{\sigma, \vec{r} \in A, \vec{\delta}_{i}}\left[c_{A, \sigma, \vec{r}}^{\dagger} c_{B, \sigma, \vec{r}+\vec{\delta}_{i}}+\text { h.c. }\right] \mathcal{P} \\
& -\mu \sum_{\sigma, o \in A, B, \vec{r}} c_{o, \sigma, \vec{r}}^{\dagger} c_{o, \sigma, \vec{r}}, \\
H_{\mathrm{K}}= & J_{\mathrm{K}} \sum_{\vec{r} \in A, \vec{\delta}_{i}} S_{\vec{r}}^{\gamma} S_{\vec{r}+\vec{\delta}_{i}}^{\gamma}, \\
H_{\mathrm{H}}= & J_{\mathrm{H}} \sum_{\vec{r} \in A, \vec{\delta} i}\left(\vec{S}_{\vec{r}} \cdot \vec{S}_{\vec{r}+\vec{\delta}_{i}}-\frac{1}{4} n_{\vec{r}} n_{\vec{r}+\vec{\delta}_{i}}\right) .
\end{aligned}
$$

The kinetic part of the Hamiltonian $H_{\text {kin }}$ describes spinindependent nearest-neighbor hopping of electrons with hopping amplitude $t_{0}$, while the chemical potential $\mu$ is adjusted to yield a charge concentration corresponding to the doping level. The Gutzwiller projection $\mathcal{P}$ enforces the constraint of no doubly occupied sites, incorporating the strong correlation effects of the Mott insulating state. Due to the two-atom unit cell of the honeycomb lattice,

\footnotetext{
${ }^{1}$ Here, we defined the exchange couplings $J_{\mathrm{H}}$ and $J_{\mathrm{K}}$ differently compared to Ref. 33 .
} 


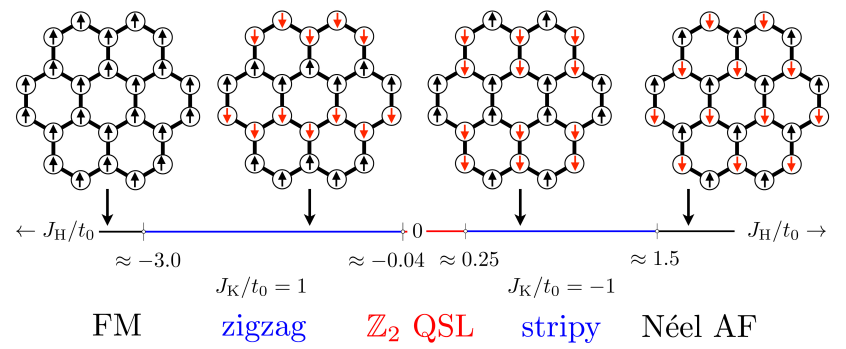

FIG. 2: Cuts in the ground-state manifold of the undoped Kitaev-Heisenberg model in the two-dimensional parameter space spanned by Kitaev $\left(J_{\mathrm{K}}\right)$ and Heisenberg $\left(J_{\mathrm{H}}\right)$ exchange couplings. In our work on the doped case, we restrict our attention to fixed $J_{\mathrm{K}}$. To facilitate a comparison to the undoped system, we chose the bare hopping as the overall energy scale and plot the ground-states for fixed Kitaev exchange as the magnitude of Heisenberg exchange is varied. For the full phase diagram in the $\left(J_{\mathrm{K}}, J_{\mathrm{H}}\right)$-plane, we refer to Ref. 22. The first row shows the magnetic ground-state patterns on the honeycomb lattice, where red and black arrows denote spin up and spin down with respect to a given quantization axis. The second row gives the corresponding parameter ranges. The numbers below indicate the critical coupling strengths, where transitions from one phase to another occur. The $\mathbb{Z}_{2}$ quantum spin liquid (which occurs for both $J_{\mathrm{K}}>0$ and $J_{\mathrm{K}}<0$ ) is destabilized by some amount of Heisenberg exchange and turns into either a magnetically ordered zigzag pattern or into an ordered stripy phase. For large ferromagnetic Heisenberg exchange, the ground-state is rendered ferromagnetic, while for large antiferromagnetic Heisenberg exchange, one finds a Néel antiferromagnet.

the kinetic term leads to a two-band description for mobile charge degrees of freedom. The sites within the twodimensional bipartite honeycomb lattice are labeled by $\vec{r}$. For fixed $\vec{r}$ in sublattice $A$, there are three nearest neighbor sites within the $B$-sublattice whose position is given by $\vec{r}+\vec{\delta}_{i}$ with $i \in\{1,2,3\}$, cf. Fig 1 . The nearestneighbor vectors $\vec{\delta}_{i}$ are given by $\vec{\delta}_{1}=\frac{\sqrt{3} a}{2} \hat{e}_{x}+\frac{a}{2} \hat{e}_{y}$, $\vec{\delta}_{2}=-\frac{\sqrt{3} a}{2} \hat{e}_{x}+\frac{a}{2} \hat{e}_{y}$ and $\vec{\delta}_{3}=-a \hat{e}_{y}$, with $a$ being the distance between two neighboring lattice sites and the vectors point from the $A$-sublattice to the $B$-sublattice. The operators $c_{o, \sigma, \vec{r}}$ and $c_{o, \sigma, \vec{r}}^{\dagger}$ describe annihilation and creation of an electron at position $\vec{r}$ in sublattice $o \in\{A, B\}$ with $\sigma=\uparrow, \downarrow$ the isospin polarization, respectively. For simplicity, we will refer to $\sigma$ as 'spin' in the remainder of the paper. We note that generally summation runs over one sublattice only (thus counting every nearest neighbor bond only once), while the sum in the local chemical-potential term runs over either sublattice $A$ or $B$, depending on whether $o=A$ or $o=B$.

Starting from a model with local interactions, within a strong-coupling expansion $\underline{43}$ virtual charge excitations above the Mott-Hubbard gap create effective spin-spin interactions. Due to large spin-orbit effects and lowsymmetry crystal fields as in the iridates, the exchange interactions are highly anisotropic. The so-called Ki- taev interaction $H_{\mathrm{K}}$ describes a bond-dependent Isinglike spin exchange, see the rightmost plaquette in Fig 1 . Its strength is described by the Kitaev coupling $J_{\mathrm{K}}$. For $i \in\{1,2,3\}$ running over the adjacent nearest-neighbor sites residing in the $B$-sublattice, $\gamma \equiv \gamma(i)$ takes on the values $\gamma \in\{x, y, z\}$. Besides the Kitaev term, the model contains an additional Heisenberg exchange $H_{\mathrm{H}}$ with Heisenberg coupling constant $J_{\mathrm{H}}$, where a nearestneighbor density-density interaction due to doping is included. We note that the presence of the densitydensity term allows to re-formulate the Heisenberg exchange solely in terms of bond-singlet operators.

The Hamiltonian Eq. (1) gives rise to a $t-J_{\mathrm{K}}-J_{\mathrm{H}}$ model description of a doped spin-orbit Mott insulator.

\section{B. Main results}

In this work, we consider the case of ferromagnetic Kitaev $\left(J_{\mathrm{K}}<0\right)$ and antiferromagnetic $\left(J_{\mathrm{H}}>0\right)$ Heisenberg exchange, as well as antiferromagnetic Kitaev $\left(J_{\mathrm{K}}>0\right)$ and ferromagnetic $\left(J_{\mathrm{H}}<0\right)$ Heisenberg exchange. While the former realizes a magnetic phase with stripy antiferromagnetic order (alternating ferromagnetic stripes that are coupled antiferromagnetically) at $\delta=0$ in the pure spin model, the latter case brings about magnetic order in a zigzag pattern (ferromagnetic zigzag chains that are coupled antiferromagnetically) $\sqrt{22}$, see Fig. 2. The $\mathbb{Z}_{2}$ quantum spin liquid exists for both ferromagnetic and antiferromagnetic Kitaev exchange for sufficiently small Heisenberg exchange strength. When both exchange couplings come with the same sign, the magnetic ordering pattern is either of ferromagnetic or Néel type.

Motivated by an estimate of the energy scale of the Kitaev exchange relative to the spin-independent nearest neighbor hopping 27 , and in order to reduce the number of parameters in the model, we restrict our attention to $\left|J_{\mathrm{K}}\right| / t_{0}=1$. As noted previously ${ }^{33}$, at fixed doping the ratio $\left|J_{K}\right| / t_{0}$ largely controls the overall scale for critical temperatures, while the ratio $\left|J_{\mathrm{K}}\right| /\left|J_{\mathrm{H}}\right|$ determines the ground-state of the doped model.

The numerical solutions to the fRG equations adapted to the Kitaev-Heisenberg model allow us to identify the leading Fermi surface instabilities of the auxiliary fermion system. We introduce the fRG equations in some detail in Sect. IV] Additionally, we can read off estimates of ordering scales or critical temperatures from the fRG flows through the critical scale $\Lambda_{c}$, at which an instability manifests as a divergence in the scale-dependent effective interaction. In the following, we will present the phase diagrams of the doped Kitaev-Heisenberg model for $J_{\mathrm{K}}<0, J_{\mathrm{H}}>0$ and $J_{\mathrm{K}}>0, J_{\mathrm{H}}<0$ as our main results. A detailed discussion of our results can be found in Sect. V] 


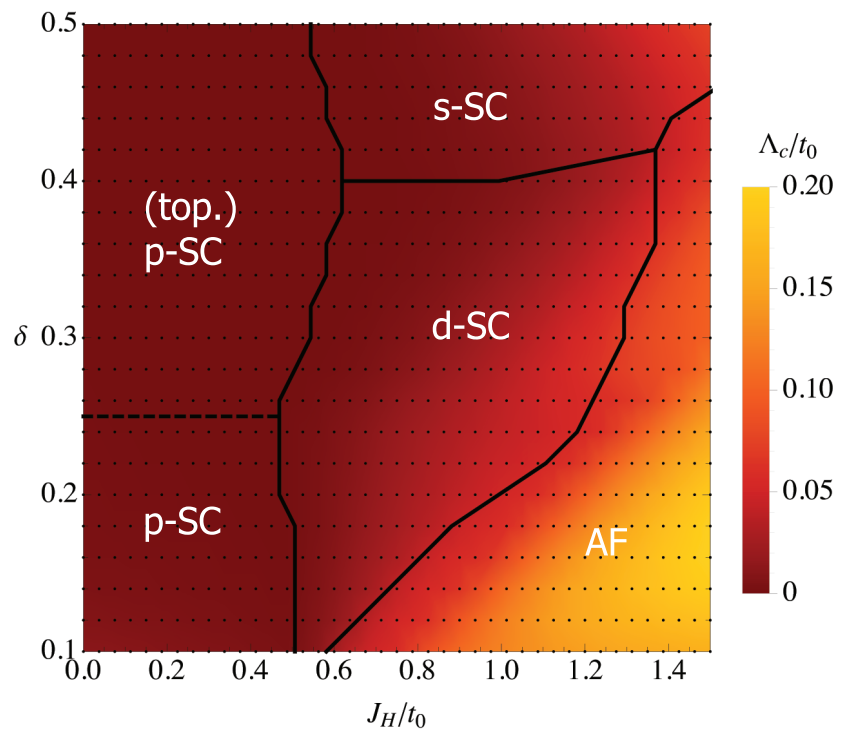

FIG. 3: The phase diagram as obtained from the numerical solution of a $(N=24)$-patching scheme with full fRG with $J_{\mathrm{K}} / t_{0}=-1$. Black dots mark the parameters for which fRGflows were evaluated. The horizontal axis gives the strength of the antiferromagnetic Heisenberg coupling $J_{\mathrm{H}}>0$ in units of the bare hopping amplitude $t_{0}$, while the doping level $\delta$ is given on the vertical axis. The colorcode describes the magnitude of the critical scale $\Lambda_{c}$ across the phase diagram. The dashed black line marks the transition to a topological oddparity pairing state across the van Hove singularity. The inclusion of particle-hole fluctuations only affects the singlet instability. The $d$-wave instability ( $d$-SC) is turned into a magnetic instability with a momentum structure corresponding to a Néel antiferromagnet (AF). For superconducting instabilities, labels in the phase diagram refer to intraband pairing symmetries. See Sect. VA for a detailed description.

\section{Ferromagnetic Kitaev $J_{\mathrm{K}}<0$, antiferromagnetic Heisenberg $J_{\mathrm{H}}>0$ exchange}

We start by considering the Kitaev-Heisenberg model with ferromagnetic Kitaev, $J_{\mathrm{K}}<0$, and antiferromagnetic Heisenberg coupling, $J_{\mathrm{H}}>0$. Fig. 3 shows the phase diagram obtained from our fRG instability analysis in the parameter space spanned by doping $\delta$ and Heisenberg exchange $J_{\mathrm{H}}$ in units of the bare hopping amplitude $t_{0}$. The Kitaev coupling is fixed to $J_{\mathrm{K}} / t_{0}=-1$ in units of the bare hopping. The general structure of the phase diagram becomes apparent already at small doping. A $p$-wave triplet superconductor is supported by ferromagnetic Kitaev exchange. The superconducting instability switches from triplet to singlet as the antiferromagnetic Heisenberg coupling increases and finally gives way to a Néel antiferromagnet, expected for large $J_{\mathrm{H}}$. Even at low doping, we find no hint of the stripy phase, one of the ground-states encountered in the undoped system, cf. Fig. 2. Although our method becomes unreliable in the limit $\delta \rightarrow 0$ due to truncation and additional approximations (see beginning of Sect. V] and Sect. VI), we in- terpret this observation as a destabilization of the stripy phase due to finite doping. For doping level $\delta>1 / 4$, we confirm topological $p$-wave phases in the doped KitaevHeisenberg model. Further, the mean-field prediction of a topological triplet $p$-wave superconductor with topologically protected Majorana modes is left untouched ${ }^{33}$ by our findings. Our results further suggest that the generation of triplet pairing instabilities in this parameter regime hinges solely on a finite ferromagnetic Kitaev exchange.

Since in the quest for realizing accessible $p$-wave superconductors, a high transition temperature into the superconducting state is desirable, let us note that we identify a window of critical scales corresponding to $p$-wave instabilities in the range $10^{-2} t_{0}$ to $10^{-4} t_{0}$ in units of the bare hopping.

\section{Antiferromagnetic Kitaev $J_{\mathrm{K}}>0$, ferromagnetic Heisenberg $J_{\mathrm{H}}<0$ exchange}

In the case of antiferromagnetic Kitaev, $J_{\mathrm{K}}>0$, and ferromagnetic Heisenberg exchange, $J_{\mathrm{H}}<0$, we again find triplet $p$-wave solutions, which are supported by both Kitaev and Heisenberg exchange. While ferromagnetic Heisenberg exchange polarizes the electronic states and eases the formation of Cooper pairs in the triplet channel, the antiferromagnetic Kitaev interaction turns out to still play a vital role in forming the instability. Indeed, for $J_{\mathrm{K}}=0$ and below a doping-dependent critical Heisenberg coupling $J_{\mathrm{H}}$, we observe no ordering tendencies down to the lowest scales accessible within our approach in neither singlet nor triplet channels with $\delta \neq 0$ (an exception is the special filling $\delta=1 / 4$, see below). The phase diagram we obtained in the plane of doping $\delta$ and Heisenberg exchange $\left|J_{\mathrm{H}}\right|$ is shown in Fig. 4 for the case of a constant Kitaev interaction $J_{K} / t_{0}=1$.

At small doping and Heisenberg exchange, we find the ground-state to realize a Néel antiferromagnet. Increasing the strength of the Heisenberg interaction while keeping the doping level low, a narrow superconducting window is sandwiched between the Néel state and a charge density wave stabilized by the density-density contribution to Eq. (1). This superconducting region increases in size as the doping grows. The ferromagnetic Heisenberg exchange naturally favors the $p$-wave triplet over singlet pairing, and we observe an extended region of triplet $p$ wave pairing throughout the phase diagram. A topological $p$-wave state hosting Majorana edge-excitations is formed for doping $\delta>1 / 4$. The range of critical scales that serves as an estimate of transition temperatures, is here given as $10^{-1} t_{0}$ to $10^{-8} t_{0}$.

For the special case of van Hove filling $\delta=1 / 4$, however, we encounter a family of instabilities not present for ferromagnetic Kitaev and antiferromagnetic Heisenberg exchange, see dashed magenta line in Fig. 4. At $\delta=1 / 4$, the Fermi surface becomes straight, leading to nesting and enhanced DOS effects. As expected, these conditions 


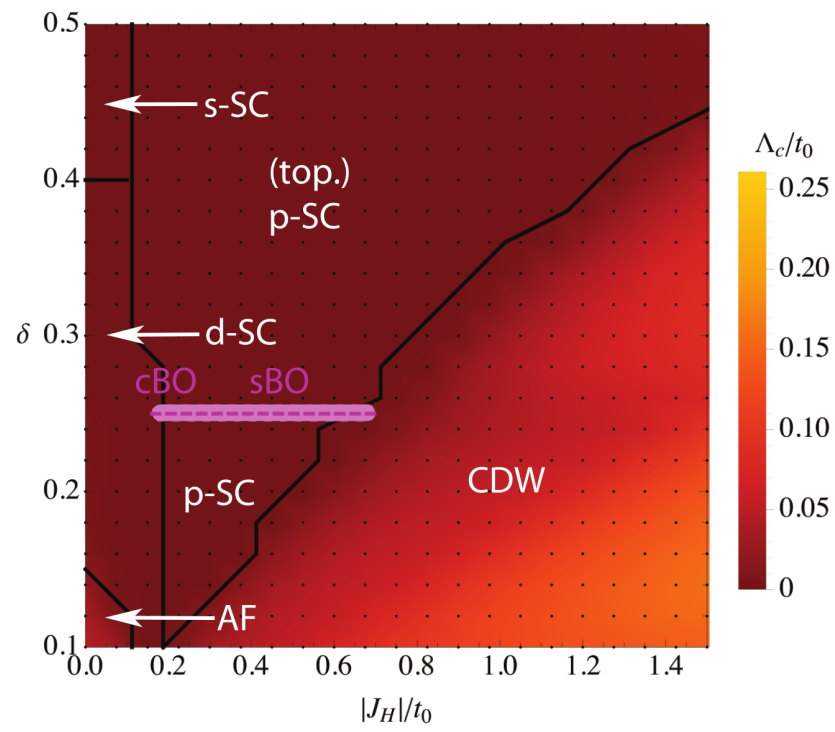

FIG. 4: The phase diagram as obtained from the numerical solution of a $(N=24)$-patching scheme with full fRG with $J_{\mathrm{K}} / t_{0}=1$. The phase boundaries were checked with a $(N=96)$-patching scheme, and only the singlet SC to triplet SC phase-boundary was mildly revised. Black dots mark the parameters for which fRG-flows were evaluated. The horizontal axis gives the strength of the ferromagnetic Heisenberg coupling $J_{\mathrm{H}}<0$ in units of the bare hopping amplitude $t_{0}$, while the doping level $\delta$ is given on the vertical axis. The colorcode describes the magnitude of the critical scale $\Lambda_{c}$ across the phase diagram. The dashed magenta line marks the van Hove singularity. The magenta shading represents the formation of charge (cBO) and spin bond-order ( $\mathrm{sBO}$ ) instabilities at van Hove filling, as obtained from a $(N=96)$-patching scheme. For the critical scales at van Hove filling, see Fig. 16 For details on bond-order, see Sect. VC The $p$-wave triplet phase appears in a parameter range where ferromagnetic exchange interactions dominate. Labels for superconducting instabilities refer to intraband pairing symmetries. For large Heisenberg exchange, the instability is of CDW type due to partice-hole fluctuations. See Sect. VB for a detailed description.

strongly enhance the tendency for particle-hole instabilities. For antiferromagnetic Kitaev and ferromagnetic Heisenberg exchange, we observe bond-order instabilities beyond a rather small critical Heisenberg coupling $\left|J_{\mathrm{H}}\right| / t_{0} \simeq 0.2$. In fact, even for $J_{\mathrm{K}}=0$, we observe the formation of bond-order instabilities at van Hove filling. The bond-order instability is leading until we hit the triplet $p$-wave/charge-density wave phase-boundary. Once the pairing neighborhood in the phase diagram has disappeared, bond-order signatures in the vertex function are rendered subleading. No leading bond-order instability is found for ferromagnetic Kitaev and antiferromagnetic Heisenberg exchange. Our results add to the current surge of unconventional bond-order instabilities with concomitant electronic dimerization in triangular lattice systems with non-trivial orbital or sublattice structure and longer-ranged interactions. We find, however, only nearest-neighbor dimerization, which rules out the possibility of a dynamically generated topological Mott insulator, that has previously been found for extended single- and multilayer honeycomb Hubbard models. Exotic properties of previously identified bond-order phases include charge fractionalization due to vortices in the Kekulé order ${ }^{44 / 45}$, valence-bond crystal states 4 , spontaneously generated current patterns $\underline{45}$ and interactiondriven emergent topological states $45 / 47$ 51. One other remarkable feature of the dimerized phase with spin bondorder of the Kitaev-Heisenberg model is the dynamical re-generation of spin-orbit coupling. A similar observation was made in the Kagome Hubbard model with fRG methods ${ }^{52}$. Interestingly, in the present case it does not lead to a gapped state, but instead we obtain a metallic state in a downfolded Brillouin zone. We note that bond order is not exclusive to systems with an underlying triangular lattice or complex orbital structure, but is also observed in square lattice systems 53 56.

\section{Outline of this Paper}

In Sect. III we derive an auxiliary fermion Hamiltonian on the honeycomb lattice that will form the input to our fRG calculations. To account for doping effects in the Kitaev-Heisenberg system, we start with a $t-J_{\mathrm{K}}-J_{\mathrm{H}}$ model. The kinetic energy will be minimally described by spin-independent hopping, assuming that high-energy spin-orbit effects are accounted for by the Kitaev exchange term. The exclusion of doubly occupied sites due to the strong interactions in the Mott insulating state, i.e., a Gutzwiller projection, is dealt with by the slaveboson method. In Sect. IIIA we discuss the slave-boson treatment of the Mott insulating state and the mean-field approximations in the bosonic sector to map the problem onto a metal of auxiliary fermions with renormalized Fermi surface coupled by exchange interactions. The resulting Hamiltonian is then split into singlet and triplet bond-operators. We then briefly recapitulate slave-boson mean-field results obtained previously by various groups in Sect. IIIB In Sect. IV] we provide the necessary background of the functional renormalization group method. It is based on the idea of the Wilsonian renormalization procedure of treating quantum corrections to the classical sector of a given theory by successively integrating out high-energy momentum shells, renormalizing the vertices of the remaining low-energy degrees of freedom. It relies on an exact hierarchy of coupled renormalization group equations for the 1-particle irreducible vertex functions. A solution of our flow equations corresponds to an unbiased resummation of 1-loop diagrams in both particle-particle and particle-hole channels. Our results are presented in detail in Sect. $\mathrm{V}$. The phase diagram for ferromagnetic Kitaev and antiferromagnetic Heisenberg exchange is discussed in Sect. VA. We first demonstrate the capability of the fRG method to reproduce mean-field results for the pairing channel in Sect. VA 1 by studying 
the effect of exclusive particle-particle fluctuations. This is done both at the level of the phase diagram and the form-factors of the superconducting order-parameters. We then move on to include particle-hole fluctuations and discuss the resulting modifications of the phase diagram in Sect. VA2 as compared to the pure particle-particle resummation in the pairing channel. In Sect. VB we flip the signs of exchange interactions and discuss the phase diagram for the doped Kitaev-Heisenberg model with antiferromagnetic Kitaev and ferromagnetic Heisenberg exchange. Sect. $\mathrm{VC}$ is devoted to the rather special filling condition $\delta=1 / 4$ with coinciding van Hove singularity and perfect Fermi surface nesting. Finally, in Sect. VI we briefly discuss our findings and discuss the validity of applying the fRG method to the $t-J_{\mathrm{K}}-J_{\mathrm{H}}$ model with strong interactions.

\section{SLAVE-BOSON FORMULATION FOR A DOPED AND FRUSTRATED MOTT INSULATOR}

In the following, we briefly describe the $U(1)$ slaveboson construction ${ }^{33}$ to deal with the Gutzwiller projection onto the Hilbert space of no doubly occupied sites. This yields an effective theory in terms of auxiliary fermionic degrees of freedom, where the bosonic charge excitations (holons) have condensed by assumption. The background holon-condensate will then give rise to an effective renormalized dispersion for the auxiliary fermions. This dispersive fermionic model will form the starting point for our fRG analysis of the doped Kitaev-Heisenberg model.

\section{A. From Kitaev-Heisenberg $t-J_{\mathrm{K}}-J_{\mathrm{H}}$ model to a $U(1)$ slave-boson model}

The local electron Hilbert-space corresponding to $c_{o, \sigma, \vec{r}}$ and $c_{o, \sigma, \vec{r}}^{\dagger}$ can be represented by fermionic $f_{o, \sigma, \vec{r}}$, bosonic holon $b_{o, \vec{r}}$ and doublon $d_{o, \vec{r}}$ degrees of freedom. This description, however, introduces unphysical states. To project the artificially enlarged Hilbert space down onto the physical states, the local constraint

$$
\sum_{\sigma} f_{o, \sigma, \vec{r}}^{\dagger} f_{o, \sigma, \vec{r}}+b_{o, \vec{r}}^{\dagger} b_{o, \vec{r}}+d_{o, \vec{r}}^{\dagger} d_{o, \vec{r}}=1
$$

needs to be enforced on the operator level. The Gutzwiller projection is now taken into account by deleting doublon operators and states from the theory.

The electron creation and annihilation operators are now to be replaced by

$$
c_{o, \sigma, \vec{r}} \rightarrow b_{o, \vec{r}}^{\dagger} f_{o, \sigma, \vec{r},}, \quad c_{o, \sigma, \vec{r}}^{\dagger} \rightarrow f_{o, \sigma, \vec{r}}^{\dagger} b_{o, \vec{r}} .
$$

Importantly, the spin operator can be written in terms of auxiliary fermions only, $\vec{S}_{\vec{r}}=f_{\vec{r}, \sigma^{\prime}}^{\dagger} \vec{\sigma}_{\sigma \sigma^{\prime}} f_{\vec{r}, \sigma^{\prime}}$, with $\vec{\sigma}=\left(\sigma_{x}, \sigma_{y}, \sigma_{z}\right)^{T}$ the vector of Pauli matrices. Assuming the holons to Bose-condense into a collective state, $\left\langle b^{\dagger} b\right\rangle=\delta$, the local constraint for the fermions becomes $\sum_{\sigma} f_{o, \sigma, \vec{r}}^{\dagger} f_{o, \sigma, \vec{r}}=1-\delta$.

The kinetic part of the Hamiltonian can now be cast in the form

$$
\begin{aligned}
H_{\mathrm{kin}}= & -t \sum_{\sigma, \vec{r} \in A, \vec{\delta}_{i}}\left[f_{A, \sigma, \vec{r}}^{\dagger} f_{B, \sigma, \vec{r}+\vec{\delta}_{i}}+\text { h.c. }\right] \\
& -\mu_{f} \sum_{\sigma, o \in A, B, \vec{r}} f_{o, \sigma, \vec{r}}^{\dagger} f_{o, \sigma, \vec{r}}
\end{aligned}
$$

with the renormalized hopping amplitude $t=t_{0} \delta$ and $\mu_{f}=\delta \mu$ the chemical potential which we adjust such that $\sum_{\sigma}\left\langle f_{o, \sigma, \vec{r}}^{\dagger} f_{o, \sigma, \vec{r}}\right\rangle=1-\delta$ is fulfilled. The constraint eliminating unphysical states is thus only included on average in this approach.

In going from the tight-binding to the Bloch representation by introducing the operators

$$
f_{o, \sigma, \vec{k}}=\frac{1}{\sqrt{\mathcal{N}}} \sum_{\vec{r}} \mathrm{e}^{\mathrm{i} \vec{k} \cdot \vec{r}} f_{o, \sigma, \vec{r}},
$$

where $\mathcal{N}$ is the number of unit cells of the honeycomb lattice, we obtain the Bloch Hamiltonian

$$
H_{\text {kin }}=-\sum_{\sigma, \vec{k}}\left(f_{A, \sigma, \vec{k}}^{\dagger}, f_{B, \sigma, \vec{k}}^{\dagger}\right)\left(\begin{array}{cc}
\mu_{f} & t d_{\vec{k}}^{*} \\
t d_{\vec{k}} & \mu_{f}
\end{array}\right)\left(\begin{array}{l}
f_{A, \sigma, \vec{k}} \\
f_{B, \sigma, \vec{k}}
\end{array}\right)
$$

with $d_{\vec{k}}=\sum_{\vec{\delta}_{i}} \mathrm{e}^{\mathrm{i} \vec{k} \cdot \vec{\delta}_{i}}$. The dispersion is analogous to electrons moving in a graphene monolayer, with the concomi$\operatorname{tant} \vec{K}$ and $\vec{K}^{\prime}$ points in the Brillouin zone.

The interaction terms quartic in the fermion operators can be expressed in terms of singlet and triplet contributions, which yields a very convenient starting point for the analysis of superconducting instabilities.

The spin-singlet operator defined on a bond connecting site $\vec{r} \in A$ to $\vec{r}+\vec{\delta}_{i} \in B$ is defined as

$$
\begin{aligned}
s_{\vec{r}, \vec{\delta}_{i}} & =f_{A, \sigma, \vec{r}}\left[\Gamma_{0}\right]_{\sigma \sigma^{\prime}} f_{B, \sigma^{\prime}, \vec{r}+\vec{\delta}_{i}} \\
& =\frac{1}{\sqrt{2}}\left(f_{A, \uparrow, \vec{r}} f_{B, \downarrow, \vec{r}+\vec{\delta}_{i}}-f_{A, \downarrow, \vec{r}} f_{B, \uparrow, \vec{r}+\vec{\delta}_{i}}\right)
\end{aligned}
$$

and correspondingly the $x, y, z$ triplet operators read

$$
\begin{aligned}
t_{\vec{r}, \vec{\delta}_{i} ; x} & =f_{A, \sigma, \vec{r}}\left[\Gamma_{x}\right]_{\sigma \sigma^{\prime}} f_{B, \sigma^{\prime}, \vec{r}+\vec{\delta}_{i}}, \\
& =\frac{1}{\sqrt{2}}\left(f_{A, \downarrow, \vec{r}} f_{B, \downarrow, \vec{r}+\vec{\delta}_{i}}-f_{A, \uparrow, \vec{r}} f_{B, \uparrow, \vec{r}+\vec{\delta}_{i}}\right) \\
t_{\vec{r}, \vec{\delta}_{i} ; y} & =f_{A, \sigma, \vec{r}}\left[\Gamma_{y}\right]_{\sigma \sigma^{\prime}} f_{B, \sigma^{\prime}, \vec{r}+\vec{\delta}_{i}} \\
& =\frac{\mathrm{i}}{\sqrt{2}}\left(f_{A, \uparrow, \vec{r}} f_{B, \uparrow, \vec{r}+\vec{\delta}_{i}}+f_{A, \downarrow, \vec{r}} f_{B, \downarrow, \vec{R}+\vec{\delta}_{i}}\right), \\
t_{\vec{r}, \vec{\delta}_{i} ; z} & =f_{A, \sigma, \vec{r}}\left[\Gamma_{z}\right]_{\sigma \sigma^{\prime}} f_{B, \sigma^{\prime}, \vec{r}+\vec{\delta}_{i}} \\
& =\frac{1}{\sqrt{2}}\left(f_{B, \uparrow, \vec{r}} f_{A, \downarrow, \vec{r}+\vec{\delta}_{i}}+f_{B, \downarrow, \vec{r}} f_{A, \uparrow, \vec{r}+\vec{\delta}_{i}}\right) .
\end{aligned}
$$

Here, we used the $\Gamma$ matrices $\Gamma_{0}=\frac{1}{\sqrt{2}} \sigma_{0} \mathrm{i} \sigma_{y}, \Gamma_{x}=$ $\frac{1}{\sqrt{2}} \sigma_{x} \mathrm{i} \sigma_{y}, \Gamma_{y}=\frac{1}{\sqrt{2}} \sigma_{y} \mathrm{i} \sigma_{y}$ and $\Gamma_{z}=\frac{1}{\sqrt{2}} \sigma_{z} \mathrm{i} \sigma_{y}$. See Appendix A 1 for explicit expressions for these matrices and 
their relation to superconducting order parameters. The interaction part of the slave-boson Hamiltonian

$$
H_{\text {slave }}=H_{\text {kin }}+H_{\text {int }}^{(\mathrm{s})}+H_{\text {int }}^{(\mathrm{t})}
$$

can now be re-cast as a sum of a singlet interaction

$$
H_{\mathrm{int}}^{(\mathrm{s})}=-\left(J_{\mathrm{H}}+\frac{J_{\mathrm{K}}}{4}\right) \sum_{\vec{r} \in A, \vec{\delta}_{i}} s_{\vec{r}_{,} \vec{\delta}_{i}}^{\dagger} s_{\vec{r}, \vec{\delta}_{i}}
$$

and a triplet interaction

$$
H_{\mathrm{int}}^{(\mathrm{t})}=-\frac{J_{\mathrm{K}}}{4} \sum_{l \in\{x, y, z\}} \sum_{\vec{r} \in A, \vec{\delta}_{i}} \zeta_{\vec{r}, \delta_{i}}^{l} t_{\vec{r}, \vec{\delta}_{i} ; l}^{\dagger} t_{\vec{r}, \vec{\delta}_{i} ; l} .
$$

Here, the index $l$ runs over the triplet components $x, y$ and $z$. The pre-factor $\zeta_{\vec{r}, \delta_{i}}^{l}$ describes a bond-dependent sign modulation of the interaction term for each triplet component: we have $\zeta_{\vec{r}, \delta_{i}}^{l}=+1$ if the bond-type $(x, y$ or $z$ ) from site $\vec{r}$ to $\vec{r}+\vec{\delta}_{i}$ coincides with the triplet component $l$. Otherwise, $\zeta_{\vec{r}, \delta_{i}}^{l}=-1$. The highly frustrated Kitaev term contains a singlet contribution, which renormalizes the singlet interaction coming from Heisenberg exchange. The contribution to the triplet channel from the Kitaev term is irreducible in the sense that it does not contain any 'hidden' singlet contributions. Thus, the singlet-triplet decomposition is unique.

We note that the interaction does not contain terms that describe the decay of a singlet state into a triplet state or vice versa. It is worth emphasizing that we model the kinetic term for the auxiliary fermions with a simple nearest-neighbor hopping, which furthermore preserves spin. Thus the only $S U(2)_{\text {spin violating contribution to }}$ the Hamiltonian comes from Kitaev exchange.

In a solid-state system with strong spin-orbit coupling, the $S U(2)_{\text {spin }}$ symmetry is locked to the point group of the lattice ${ }^{34 \mid 57}$, i.e., only the simultaneous application of point-group transformations and the corresponding representation of the point-group operations on the spin degrees of freedom leave the Hamiltonian invariant. In, e.g., the iridates the Kitaev term arises precisely due to the presence of strong spin-orbit coupling. As noted previously $\sqrt{34}$, it is thus natural that the symmetry of the Kitaev term involves simultaneous transformation of both spin and lattice (or wavevector). The relevant symmetry transformations in the case of the Kitaev term (with the same coupling $J_{\mathrm{K}}$ on nearest-neighbor bonds) acting on the lattice can be understood as a $2 \pi / 3$ rotation around the center of a honeycomb hexagon. To maintain invariance of the Kitaev Hamiltonian, spins have to be rotated by the same angle around the axis $\hat{n}=\frac{1}{\sqrt{3}}(1,1,1)^{T}$, where the coordinate system corresponds to an embedding of a honeycomb layer into a 3D cubic lattice ${ }^{34}$. See Appendix A 4 for further details.

\section{B. Comparison with mean-field theory}

The phase diagram of the doped Kitaev-Heisenberg model has previously been studied within different slave- boson formulations. If quantum fluctuations were treated exactly in these different formulations, they should yield equivalent results. In practice, however, the emergent gauge fields in slave-boson approaches for strongly correlated lattice fermions are treated in a mean-field approximation. The local Hilbert-space constraint is realized only on average. While this is also true in our slave-boson formulation of the $t-J_{\mathrm{K}}-J_{\mathrm{H}}$ model, where the holoncondensation is built into the theory in mean-field fashion, the fermionic fRG takes into account the fermionic fluctuations in all channels in an otherwise unbiased way. To allow for a systematic and self-contained comparison of our fRG results to mean-field studies reported in the literature, we briefly summarize recent findings.

\section{U(1) Slave-boson mean-field theory}

A previous self-consistent mean-field study ${ }^{33}$ of the doped Kitaev-Heisenberg model taking into account only superconducting order-parameters demonstrated a rich phase diagram upon doping charge carriers into the $\mathbb{Z}_{2}$ quantum spin liquid (QSL) and the Mott insulating stripy phase. For ferromagnetic Kitaev coupling, $J_{\mathrm{K}}<0$, the QSL state at zero doping is stable with respect to Heisenberg-type perturbations for $\left|J_{\mathrm{H}}\right|<\left|J_{\mathrm{K}}\right| / 8$, cf. Ref. 27. Keeping the value of the Kitaev exchange coupling fixed for the undoped system, the stripy phase is realized for antiferromagnetic Heisenberg exchange coupling, $J_{\mathrm{H}}>0$, with $J_{\mathrm{H}} \simeq 0.25, \ldots, 1.5$. At finite doping, $\delta \gtrsim 0.1$, for small antiferromagnetic Heisenberg coupling and dominating ferromagnetic Kitaev coupling, $\left|J_{\mathrm{K}}\right| / 2>J_{\mathrm{H}}$, a time-reversal invariant $p$-wave state $(p$ $\mathrm{SC})$ was found. Upon increasing doping in this regime, a topological phase transition to a topological pairing state with $p$-wave symmetry in the triplet channel was obtained ${ }^{33}$ in the vicinity of van Hove filling. This state is stable to interband pairing correlations and was recently shown to be robust also against weak non-magnetic disorder ${ }^{36}$. Its topological properties can be characterized by a non-trivial topological $\mathbb{Z}_{2}$-invariant ${ }^{33 \mid 36}$ from which we can infer that it falls into the DIII symmetry class of the Altland-Zirnbauer classification 8 .

For antiferromagnetic Heisenberg coupling $J_{\mathrm{H}} \gtrsim$ $\left|J_{\mathrm{K}}\right| / 2$ at small doping and ferromagnetic Kitaev coupling, the leading pairing correlations occur in the singlet channel with intraband $d$-wave symmetry. In the large $J_{\mathrm{H}}$ regime, an extended singlet $s$-wave state was reported.

\section{2. $S U(2)$ Slave-boson mean-field theory}

Within an $S U(2)$ formulation 34 and a specific meanfield Ansatz that reproduces the $\mathbb{Z}_{2}$ quantum spin liquid in the $\delta=0$ Kitaev limit $J_{\mathrm{H}}=0$, a time-reversal symmetry breaking $p$-wave state in the triplet channel $\left(p-\mathrm{SC}_{1}\right)$ was found upon doping the stripy phase. Interestingly, it 
supports chiral edge modes and localized Majorana states in the low-doping regime. The physics of this state was argued to be dominated by its vicinity to the QSL at $J_{\mathrm{H}}=0$, while for increased doping a first order transition to a BCS like state $\left(p-\mathrm{SC}_{2}\right)$ occurs.

A further extensive mean-field study ${ }^{35}$ for the doped Kitaev-Heisenberg model utilizing the $S U(2)$ formulation with an Ansatz that includes pairing and magnetic order-parameters supports the previous findings. In both mean-field studies for ferromagnetic $J_{\mathrm{K}}$ and antiferromagnetic $J_{\mathrm{H}}$ couplings, however, time-reversal breaking and time-reversal invariant $p$-wave states are reported as (almost) energetically degenerate for the $p-\mathrm{SC}_{2}$ state. In the time-reversal invariant case, the $p-\mathrm{SC}_{2}$ state obtained from the $S U(2)$ formulation coincides with the $p$-SC state from the $U(1)$ slave-boson theory. For large $\left|J_{\mathrm{H}}\right|$, again singlet instabilities are obtained, with pairing symmetry changing from $d$ - to $s$-wave upon increasing the doping level.

For antiferromagnetic $J_{\mathrm{K}}$ and ferromagnetic $J_{\mathrm{H}}$, a $p$ $\mathrm{SC}_{1}$ phase that extends to large doping was reported. Roughly, for dominating antiferromagnetic Kitaev coupling, a $d$-wave singlet solution was obtained, with a transition to a $p-\mathrm{SC}_{2}$ phase upon increasing $J_{\mathrm{H}}$ and/or doping. For $J_{\mathrm{H}} \gg\left|J_{\mathrm{K}}\right|$, all pairing correlations disappear and a ferromagnetic ordering emerges. Mean-field Ansätze other than superconducting and ferromagnetic were not considered.

\section{FRG METHOD}

We employ a functional renormalization group (fRG) approach for the one-particle-irreducible (1PI) vertices with a momentum cutoff. For a recent review on the fRG method, see Refs. 42 158. The actual fRG calculation is performed in the band-basis in which the quadratic part of the fermion Hamiltonian is diagonal. The free Hamiltonian can be diagonalized by a unitary transformation of the form

$$
f_{b, \sigma, \vec{k}}=\sum_{o} u_{b o, \vec{k}} f_{o, \sigma, \vec{k}}, \quad f_{b, \sigma, \vec{k}}^{\dagger}=\sum_{o} u_{b o, \vec{k}}^{*} f_{o, \sigma, \vec{k}}^{\dagger},
$$

where $o \in\{A, B\}$ labels the two sublattices and the index $b$ denotes the corresponding bands. This transformation also affects the bare interaction vertex and leads to socalled orbital make-up. Since we do not consider mixing of spin states by the kinetic term of the Hamiltonian, this unitary transformation does not involve spin projection. In band representation, the propagator is also diagonal, with diagonal entries encoding the dispersion $\epsilon(\vec{k}, b)$ of the various bands labeled by $b$. In the standard 1PI fRGscheme we employ here, an infrared regulator with energy scale $\Lambda$ is introduced into the bare propagator function in band representation $G_{0}\left(\xi, \xi^{\prime}\right) \sim \delta_{\xi, \xi^{\prime}}$, where the label $\xi=(\sigma, b, \omega, \vec{k})$ collects spin projection $\sigma$, band index $b$, frequency $\omega$ and Bloch momentum $\vec{k}$. We thus replace

$$
G_{0}(b, \omega, \vec{k}) \rightarrow G_{0}^{\Lambda}(b, \omega, \vec{k})=\frac{C^{\Lambda}[\epsilon(\vec{k}, b)]}{i \omega-\epsilon(\vec{k}, b)} .
$$

As the spin quantum number $\sigma$ carried by the auxiliary fermion degrees of freedom is conserved by the kinetic part of the Hamiltonian Eq. (14) the free propagator is diagonal also in spin indices. The cutoff function is chosen to enforce an energy cutoff, which regularizes the free Green's function by suppressing the modes with band energy below the scale $\Lambda$,

$$
C^{\Lambda}[\epsilon(\vec{k}, b)] \approx \Theta(|\epsilon(\vec{k}, b)|-\Lambda) .
$$

For better numerical feasibility the step function is slightly softened in the actual implementation. With this modified scale-dependent propagator, we can define the scale-dependent effective action $\Gamma^{\Lambda}$ as the Legendre transform of the generating functional $\mathcal{G}^{\Lambda}$ for correlation functions, cf. Refs. 42/59, The RG flow of $\Gamma^{\Lambda}$ is generated upon variation of $\Lambda$. By integrating the flow down from an initial scale $\Lambda_{0}$ one smoothly interpolates between the bare action of the system and the effective action at low energy.

\section{A. Flow equations for $S U(2)_{\text {spin }}$ non-invariant systems}

While the $U(1)$ slave-boson model Eq. (14) is equipped with global $U(1)$ symmetry in the fermion sector even after the bosonic holons have condensed, there is no $S U(2)_{\text {spin }}$ symmetry present as for electronic systems without spin-orbit coupling. Since the interactions do not conserve the spin quantum number carried by the fermions, the four-point vertex will also depend on the specific spin configuration of incoming and outgoing states. Without any further symmetry constraints, this leads to a total of $16=2^{4}$ independent coupling functions, one for each possible spin configuration. For the Hamiltonian in Eq. (14), however, it suffices to consider only four vertex functions. These are simply given by singlet-singlet and triplet-triplet interactions. The renormalization group flow preserves this property, i.e., if it is present in the initial condition, singlet-triplet mixing terms will never be generated during the RG evolution. ${ }^{2}$

This allows us to factor out the spin-indices from the vertex functions. To this end we introduce the shorthand notation $\xi=(\sigma, \tilde{\xi})$ for the set of quantum numbers, where $\tilde{\xi}=(o, \omega, \vec{k})$ in orbital/sublattice representation and $\tilde{\xi}=(b, \omega, \vec{k})$ in band representation. After

\footnotetext{
2 This property hinges on the $S U(2)_{\text {spin }}$ invariance of the kinetic Hamiltonian. Generally, the fRG-flow is expected to leave the manifold spanned by the singlet and triplet vertex functions, if we were to include spin dependent hopping processes. Then the full space of spin configurations needs to be resolved.
} 
factorization, the flow equations can be formulated for only four vertex functions depending on the quantum numbers $\tilde{\xi}$, exclusively. With these premises, the scaledependent coupling function $V^{\Lambda}=V^{\Lambda}\left(\xi_{1}, \xi_{2}, \xi_{3}, \xi_{4}\right)$ can be expanded in terms of the singlet vertex function, $V^{(\mathrm{s})}=V^{(\mathrm{s})}\left(\tilde{\xi}_{1}, \tilde{\xi}_{2}, \tilde{\xi}_{3}, \tilde{\xi}_{4}\right)$, and the triplet vertex functions, $V_{l}^{(\mathrm{t})}=V_{l}^{(\mathrm{t})}\left(\tilde{\xi}_{1}, \tilde{\xi}_{2}, \tilde{\xi}_{3}, \tilde{\xi}_{4}\right)$, as

$$
\begin{aligned}
V^{\Lambda}=- & V^{(\mathrm{s})}\left[\Gamma_{0}^{\dagger}\right]_{\sigma_{1} \sigma_{2}}\left[\Gamma_{0}\right]_{\sigma_{3} \sigma_{4}} \\
& +\sum_{l \in\{x, y, z\}} V_{l}^{(\mathrm{t})}\left[\Gamma_{l}^{\dagger}\right]_{\sigma_{1} \sigma_{2}}\left[\Gamma_{l}\right]_{\sigma_{3} \sigma_{4}}
\end{aligned}
$$

The projection onto scale-dependent singlet $V^{(\mathrm{s})}$ and triplet $V_{l}^{(\mathrm{t})}$ vertex functions can be facilitated with the orthogonality properties of the $\Gamma$ matrices, see Appendix A 1. effectively tracing out spin quantum numbers $\sigma_{i}$, $i=1, \ldots, 4$.

The singlet vertex function $V^{(\mathrm{s})}$ is fully symmetric with respect to exchanging $\tilde{\xi}_{1} \leftrightarrow \tilde{\xi}_{2}$ and $\tilde{\xi}_{3} \leftrightarrow \tilde{\xi}_{4}$. The antisymmetric spin matrix $\Gamma_{0}$ ensures overall antisymmetry under these exchange operations, as required by a fermionic 4-point function. Correspondingly, the triplet vertex functions $V_{l}^{(\mathrm{t})}$ are antisymmetric under these exchange operations with symmetric spin matrices $\Gamma_{l}$.

Employing the flow equations appropriate for global $U(1)$ symmetry $\underline{60}$, we find for the singlet evolution

$$
\frac{d}{d \Lambda} V^{(\mathrm{s})}=\phi_{\mathrm{pp}}^{(\mathrm{s})}+\phi_{\mathrm{ph}, \mathrm{cr}}^{(\mathrm{s})}+\phi_{\mathrm{ph}, \mathrm{d}}^{(\mathrm{s})},
$$

where $\phi_{\mathrm{pp}}^{(\mathrm{s})}, \phi_{\mathrm{ph}, \mathrm{d}}^{(\mathrm{s})}$ and $\phi_{\mathrm{ph}, \mathrm{cr}}^{(\mathrm{s})}$ denote the particle-particle, direct particle-hole and crossed particle-hole RG contributions to the singlet channel. The triplet evolutions are found along the same lines as

$$
\frac{d}{d \Lambda} V_{l}^{(\mathrm{t})}=\phi_{\mathrm{pp} ; l}^{(\mathrm{t})}+\phi_{\mathrm{ph}, \mathrm{cr} ; l}^{(\mathrm{t})}-\phi_{\mathrm{ph}, \mathrm{d} ; l}^{(\mathrm{t})}, \quad l \in\{x, y, z\},
$$

with the corresponding RG contributions for the three triplet channels. The scale-dependent bubble contributions appearing on the right hand sides of Eq. (21) and Eq. (22) are in turn quadratic functionals of $V^{(\mathrm{s})}$ and $V_{l}^{(\mathrm{t})}$. The explicit expressions are summarized in Appendix A 3 From these it follows, that singlet and triplet channel exert a mutual influence only through particlehole fluctuations. For a diagrammatic representation of the flow equations Eq. 211 and Eq. (22) for singlet and triplet vertices, see Fig. 5 . Neglecting both direct and crossed particle-hole contributions to the RG evolutions of singlet and triplet coupling functions, Eq. (21) and Eq. (22) decouple so that singlet and triplet vertex functions evolve independently. This decoupling of singlet and triplet channels is also found in BCS-like mean-field theory from a linearized gap-equation. We thus expect that the inclusion of only the particle-particle bubbles $\phi_{\mathrm{pp}}^{(\mathrm{s})}$ and $\phi_{\mathrm{pp} ; l}^{(\mathrm{t})}$ as driving forces in the flow equations yields a resummation that reproduces mean-field results. Details are discussed in Sect. VA
We note there is a difference in sign between crossed and direct particle-hole contributions in the singlet Eq. 21) and triplet Eq. 222 flow equations. This sign reflects the exchange symmetries of the various vertex functions, such that the incremental changes $d V^{(\mathrm{s})}$ and $d V_{l}^{(\mathrm{t})}$ come with the correct (anti-)symmetrization, since neither crossed nor direct particle-hole bubbles have the symmetry property on their own.

\section{B. Approximations and numerical implementation}

In order to limit the numerical effort we employ a number of approximations. First, the hierarchy of flowing vertex functions is truncated after the four-point (twoparticle interaction) vertex. Second, we employ the static approximation, i.e., we neglect the frequency dependence of vertex functions, by setting all external frequencies to zero, as we are interested in ground-state properties. Third, self-energy corrections are neglected. This approximate fRG scheme then amounts to an infinite-order summation of one-loop particle-particle and particle-hole terms of second order in the effective interactions. It allows for an unbiased investigation of the competition between various correlations, by analyzing the components of $V^{(\mathrm{s})}$ and $V_{l}^{(\mathrm{t})}$ that create instabilities by growing large at a critical scale $\Lambda_{c}{ }^{42}$. From the evolving pronounced momentum structure one can then infer the leading ordering tendencies. With the approximations mentioned above, this procedure is well-controlled for small interactions. At intermediate interaction strengths we still expect to obtain reasonable results and it was recently shown that the fRG-flow produces sensible results even in proximity to the singularity ${ }^{61}$. In any case, the fRG takes into account effects beyond mean-field and random phase approximations. This way, the fRG represents an alternative to the inclusion of gauge fluctuations in the mean field theories. The wavevector dependence of the interaction vertices is simplified by a discretization the $N$-patch scheme - that resolves the angular dependence along the Fermi surface for a given chemical potential. The Brillouin zone (BZ) is divided into $N$ patches with constant wavevector dependence within one patch, so that the coupling function has to be calculated for only one representative momentum in each patch. The representative momenta for the patches are chosen to lie close to the Fermi level. The patching scheme is shown in Fig. 6 for $N=24$. Calculations were performed for different but fixed angular resolution with $N=24$ and $N=48$ as well as $N=96$ to check the reliability of the results with respect to higher resolution. The vertex functions further depend on sublattice or band labels. Since overall momentum conservation leaves only three independent wavevectors in the BZ, a single vertex function is approximated by a $2^{4} \times N^{3}$ component object. In total, we thus obtain $4 \times 2^{4} \times N^{3}$ coupled differential equations for the approximated singlet and triplet vertex functions. Exploiting the implications of residual rotational symme- 

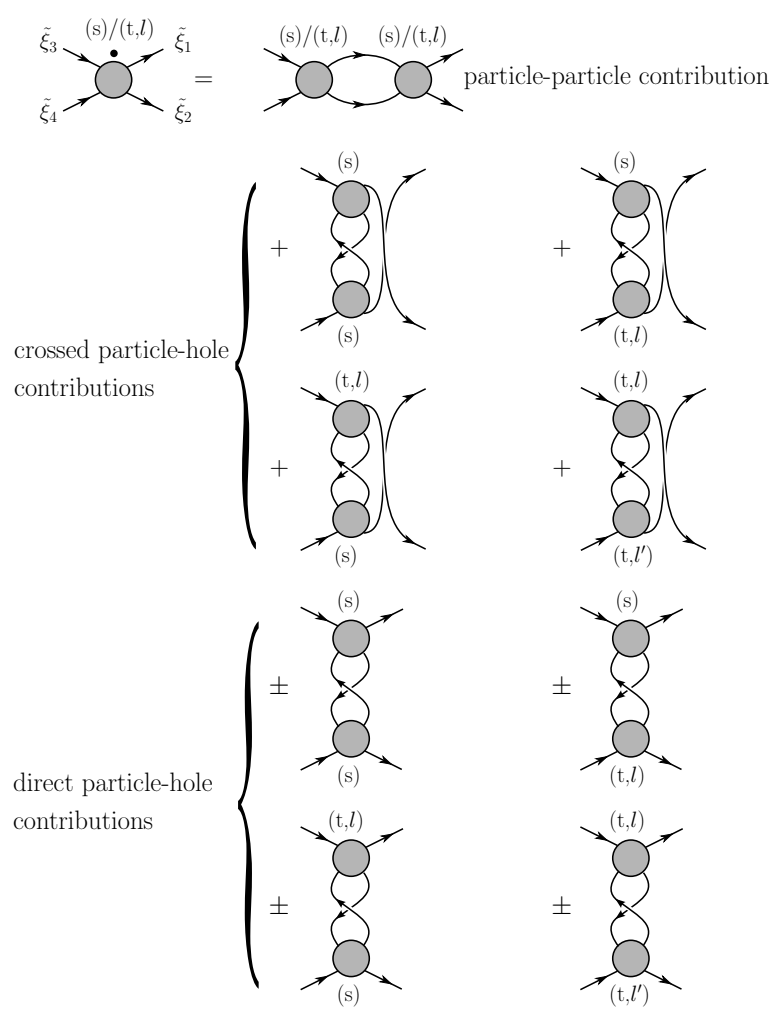

FIG. 5: Diagrammatic representation of flow Eq. (21) and Eq. 222. The dot on the l.h.s of the equation denotes the scale derivative $d / d \Lambda$. In the loops, one line always corresponds to a bare regularized propagator $G_{0}^{\Lambda}$, while the other one corresponds to a so-called single scale propagator $S^{\Lambda}=d / d \Lambda G_{0}^{\Lambda}$. The labels $(\mathrm{s})$ and $(\mathrm{t}, l)$ distinguish singlet and triplet vertices. The first contribution to the flow is the respective particleparticle diagram $\phi_{\mathrm{pp}}^{(\mathrm{s})}, \phi_{\mathrm{pp} ; l}^{(\mathrm{t})}$ that does not couple singlet and triplet channels. The remaining two sets of diagrams correspond to the crossed $\left(\phi_{\mathrm{ph}, \mathrm{cr}}^{(\mathrm{s})}, \phi_{\mathrm{ph}, \mathrm{cr} ; l}^{(\mathrm{t})}\right)$ and direct particle-hole bubbles $\left(\phi_{\mathrm{ph}, \mathrm{d}}^{(\mathrm{s})}, \phi_{\mathrm{ph}, \mathrm{d} ; l, l}^{(\mathrm{t})}\right)$. In the direct particle-hole diagrams the positive (negative) signs refer to the contributions to the flow of the singlet (triplet) vertex. See also Appendix A 3 for details on the different sign structures for singlet and triplet flow equations.

try as outlined above (see also Appendix A 4 for vertex reconstruction in the triplet channel, this number can be reduced by a factor of 2 .

\section{ORDERING TENDENCIES FROM FUNCTIONAL RG FLOWS}

We start the fRG flow at the initial scale $\Lambda_{0}$ which we choose as the largest distance in energy from the location of the Fermi surface to the lower and top band edges of valence and conduction bands, respectively. By solving the flow equations numerically, we successively integrate out all modes of these bands in energy shells with support peaked around the RG-scale $\Lambda$. In the case of an

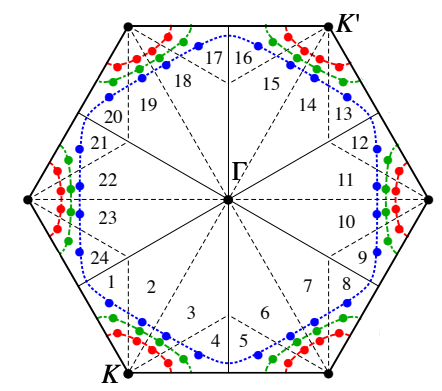

FIG. 6: Patching of the Brillouin zone (BZ) with $N=24$ for different doping levels. For $\mu \neq 0$ the representative patch momenta (colored dots) move toward the $\Gamma$-point in the BZ center. At $|\mu|=t_{0}$ the Fermi surface segments become 'straight', i.e., perfect nesting is realized, which is reflected in a van Hove singularity in the density of states. For $|\mu| \gtrsim t_{0}$ the actual implementation of the patching needs to be slightly modified. The red, green and blue lines correspond to the Fermi surfaces for doping $\delta \approx 0.08,0.14,0.27$, respectively.

instability, some components of the scale-dependent effective interaction vertices become large and eventually diverge at a critical scale $\Lambda_{c}>0$. Since the flow needs to be stopped at a scale $\Lambda^{*} \lesssim \Lambda_{c}$, we take as a stopping criterion the condition that the absolute value of one of the vertex functions exceeds a value of the order of 100 times the bare bandwidth $t_{0}$. We further assume that $\Lambda^{*} \simeq \Lambda_{c}$. The precise choice for the stopping criterion affects the extracted value for the critical scale very mildly, as the couplings grow very fast in the vicinity of the divergence.

In our analysis, we kept the value of the bare hopping $t_{0}$ fixed, and also fixed the value for the Kitaev coupling $J_{K}$, while doping $\delta$ and Heisenberg coupling $J_{\mathrm{H}}$ are varied.

To elucidate the role of holon-condensation on the system parameters, cf. Eq. (7), we consider the auxiliary fermion Hamiltonian Eq. (14), and its corresponding partition function $Z(\beta)=\operatorname{Tr}^{-\beta H_{\text {slave }}}$. While doping levels $\delta<1$ reduce the bandwidth of the fermion system, we can equivalently view this as a renormalization of the bare exchange interactions. Since the partition function $Z(\beta)$ is invariant upon rescaling temperature as $\beta \rightarrow \beta \delta$ and at the same time rescaling the Hamiltonian $H_{\text {slave }} \rightarrow H_{\text {slave }}^{\prime}=H_{\text {slave }} / \delta$, the ground-state properties of $H_{\text {slave }}$ in the limit $\beta \rightarrow \infty$ can be extracted from $H_{\text {slave }}^{\prime}$.

In total, this corresponds to rescaling hopping amplitude, chemical potential and couplings as

$$
t \rightarrow \frac{t}{\delta}=t_{0}, \mu_{f} \rightarrow \frac{\mu_{f}}{\delta}=\mu, J_{\mathrm{H}} \rightarrow \frac{J_{\mathrm{H}}}{\delta}, J_{\mathrm{K}} \rightarrow \frac{J_{\mathrm{K}}}{\delta} .
$$

The rescaling entails large absolute values for the vertex functions already in the initial condition at least in the low-doping regime $\delta \simeq 0.1$. While we can conveniently keep the kinetic energy scale at $t_{0}$, we cannot set the bar for the stopping criterion too low on the vertex functions to allow - at least - for a sizable evolution along the $\Lambda$ direction. From these considerations it is also immediately obvious that within our slave-boson approach and 
the employed approximations to the exact hierarchy of flow equations we cannot describe the magnetic instabilities of the Mott insulator as $\delta \rightarrow 0$. Including the flowing self-energy $\Sigma^{\Lambda}$ and keeping frequency dependence in the flows for self-energy and effective interaction vertex, we could expect to bridge the description to the Mott insulating state, cf. Sect. VI and Refs. 26 2962.

A divergence in the interaction vertex can be considered as an artefact of our truncation, which as it stands completely neglects self-energy feedback. Here, we thus restrict ourselves to an analysis of leading ordering tendencies at finite doping. The pronounced momentum structure of a vertex function close to the critical scale $\Lambda_{c}$ can be used to extract an effective Hamiltonian for the low-energy degrees of freedom, which can in principle be decoupled by a suitable Hubbard-Stratonovich field that is then dealt with on a mean-field level. This is used to determine the order parameter corresponding to the leading instability. Furthermore, the scale $\Lambda_{c}$ can be interpreted as an estimate for ordering temperatures, if ordering is allowed, or at least as the temperature below which the dominant correlations should be clearly observable.

\section{A. Doping QSL and stripy phase - FM Kitaev and AF Heisenberg exchange}

We first consider the case of a ferromagnetic Kitaev, $J_{\mathrm{K}}<0$, and antiferromagnetic Heisenberg coupling, $J_{\mathrm{H}}>0$. At doping level $\delta=0$, there exists an extended region in the space of couplings $J_{\mathrm{H}}, J_{\mathrm{K}}$ where the stripy phase is realized as the magnetically ordered ground-state of the strongly correlated spin-orbit Mott insulator. In the following, we will fix the ferromagnetic Kitaev coupling as $J_{\mathrm{K}} / t_{0}=-1$. The range of Heisenberg couplings we focus on is given by $J_{\mathrm{H}} / t_{0} \in[0,1.5]$. This parameter range includes the full extent of the stripy phase at $\delta=0$, and also for small $J_{\mathrm{H}}$, the dominating Kitaev term is responsible for the realization of the quantum spin liquid phase (QSL). However, here we focus on a doping regime $\delta \in[0.1,0.5]$, where the effects of the proximity to the QSL phase are not visible any more. We thus cannot observe a signature of the $p-\mathrm{SC}_{1}$ state ${ }^{34 \mid 35}$, cf. Sect. IIIB. Slave-boson mean-field studies connecting to the Kitaev-limit as $\delta \rightarrow 0$ suggest $[3435$ that holon-condensation sets in rapidly at $J_{\mathrm{H}} \neq 0$ as $\delta$ is increased from 0 to a small but finite value. Although this seems to render our Hamiltonian a sensible starting point from the point of view of mean-field theory, the small renormalized band-width $\sim t$ yields huge rescaled couplings, turning the fRG-flow unreliable. In the doping regime $\delta<0.1$, a pure particle-particle resummation detects no sign of a first order transition between two different triplet $p$-wave phases. While upon the inclusion of partice-hole fluctuations the superconductivity seems to disappear, the divergent vertex functions do not yield a clear picture as to what kind of instability is actually

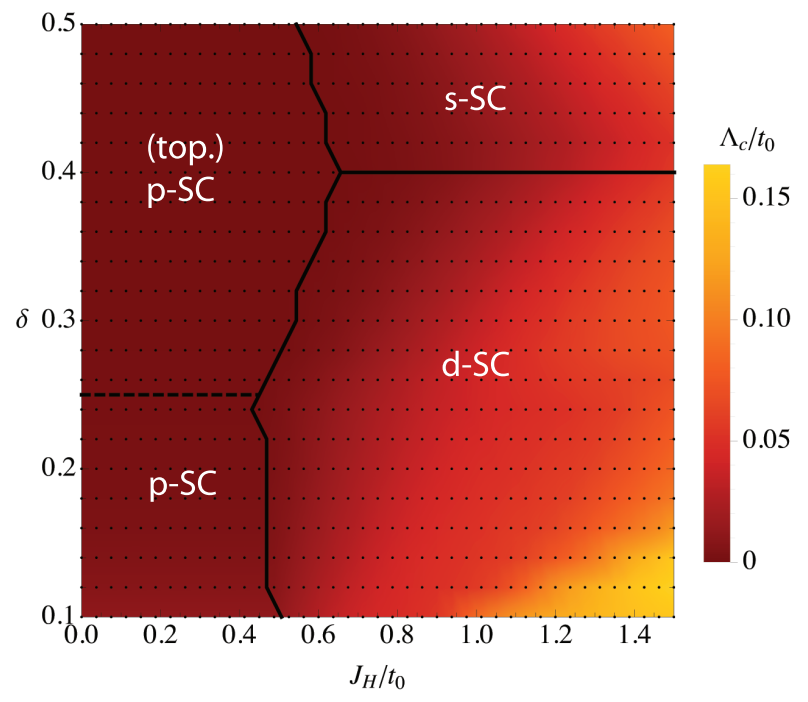

FIG. 7: The phase diagram as obtained from the numerical solution of a $(N=24)$-patching scheme with only particleparticle bubbles with $J_{\mathrm{K}} / t_{0}=-1$. Black dots mark the parameters for which fRG-flows were evaluated. The horizontal axis gives the strength of the antiferromagnetic Heisenberg coupling $J_{\mathrm{H}}>0$ in units of the bare hopping amplitude $t_{0}$, while the doping level $\delta$ is given on the vertical axis. The colorcode describes the magnitude of the critical scale $\Lambda_{c}$ in units of the bare hopping $t_{0}$ across the phase diagram. The dashed black line marks the transition to a topological oddparity pairing state across the van Hove singularity. In this approximation we obtain several superconducting instabilities, where labels refer to intraband pairing symmetries. See the main text for a detailed description.

realized. As a consistency check on our flow equations, we first completely neglect crossed and direct particlehole bubbles in the flow. From a diagrammatic perspective, the numerical solution of the flow equations is expected to reproduce the results of a mean-field analysis of the slave-boson Hamiltonian with only superconducting order parameters. Second, we obtain the phase diagram for the doped stripy phase with particle-particle and particle-hole bubbles included on equal footing in the flow equations.

\section{Resummation in particle-particle channel}

As already mentioned in Sect. IV, only particle-hole fluctuations couple singlet and triplet vertex functions among each other. Neglecting particle-hole bubbles amounts to taking into account only the first diagram in Fig. 5 in the flow equation. We find, that the leading instability is essentially determined by the structure of the initial condition for the flow equation. For a dominating singlet vertex, the flow leads to an increase in the singlet channel, where also the dominating pairing symmetry of the initial condition is enhanced. The amplitude of the subleading pairing solutions is not substantially increased 

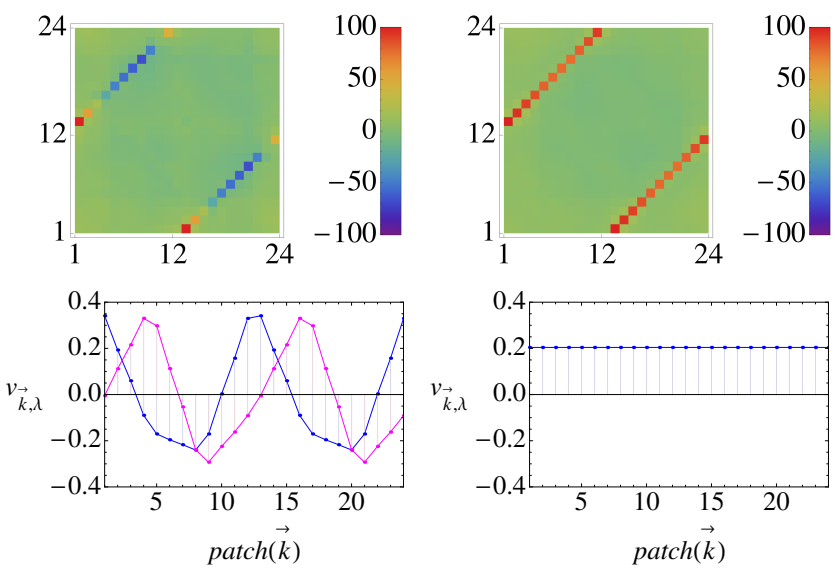

FIG. 8: The upper left panel shows the intraband component of the divergent singlet vertex function for $\delta=0.2$ and $J_{\mathrm{H}} / t_{0}=0.9$, where the patch numbers corresponding to patch momentum $\vec{k}_{1}$ are given on the ordinate and $\vec{k}_{2}$ on the abscissa. The remaining free momentum $\vec{k}_{3}$ is fixed to the first patch, cf. Fig. 6. The divergent momentum structure corresponds to a $d$-wave instability. The upper right panel shows the intraband component of the divergent singlet vertex function for $\delta=0.44$ and $J_{\mathrm{H}} / t_{0}=1.31$, with a momentum structure yielding an $s$-wave instability. In the lower left panel we display the normalized amplitude of the two degenerate eigenvectors with $d$-wave symmetry of the singlet pair-scattering amplitude along the Fermi surface. The patch number is enumerated on the abscissa. The lower right panel shows the corresponding eigenvector with $s$-wave symmetry.

by the fRG-evolution. For a superconducting instability, the associated spin-structure of the leading pairing correlations can be immediately inferred from whether the singlet vertex $V^{(\mathrm{s})}$ or the triplet vertices $V_{l}^{(\mathrm{t})}$ diverge. Due to the residual rotational symmetry of the KitaevHeisenberg model (see Sect. IV and Appendix A 4), the three triplet vertex functions are bound to diverge simultaneously. This also leads to a degeneracy for the pairing solutions for the $\vec{d}$-vector describing the structure of the corresponding Cooper pair. In order to obtain such information from the vertex functions, we extract the pair scattering amplitudes in singlet and triplet channels as

$$
\begin{aligned}
V^{(\mathrm{s})}\left(\vec{k}, \overrightarrow{k^{\prime}}\right) & \equiv V^{(\mathrm{s})}\left(\vec{k},-\vec{k}, \vec{k}^{\prime},-\vec{k}^{\prime}\right), \\
V_{l}^{(\mathrm{t})}\left(\vec{k}, \overrightarrow{k^{\prime}}\right) & \equiv V_{l}^{(\mathrm{t})}\left(\vec{k},-\vec{k}, \vec{k}^{\prime},-\vec{k}^{\prime}\right),
\end{aligned}
$$

where for brevity we suppressed sublattice or band labels. Since our discretization of the Brillouin zone employs a total of $N$ representative patch momenta, the pair-scattering amplitudes can be treated as $N \times N$ matrices. Diagonalization of $V^{(\mathrm{s})}\left(\vec{k}, \overrightarrow{k^{\prime}}\right)$ or $V_{l}^{(\mathrm{t})}\left(\vec{k}, \overrightarrow{k^{\prime}}\right)$ and determination of the eigenvectors $v_{\vec{k}, \lambda}$ corresponding to the eigenvalues $\lambda$ unveils the leading and subleading pairing instabilities 63 . The 2-particle contributions to the effective action $\Gamma^{\Lambda_{c}}$ at the critical scale $\Lambda_{c}$ determined by the leading instability (i.e. the eigenvalue $\lambda$ with largest
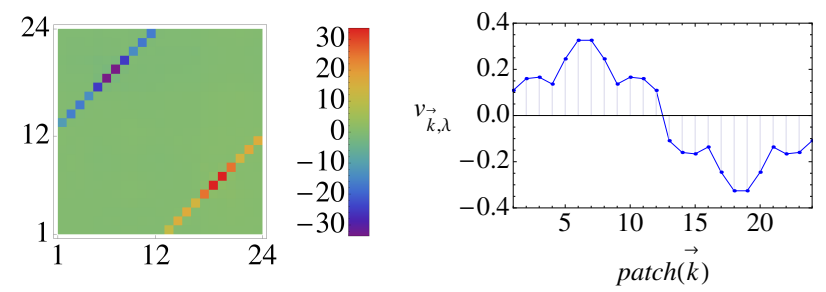

FIG. 9: The left panel shows the intraband component of the divergent triplet vertex function for $\delta=0.3$ and $J_{\mathrm{H}} / t_{0}=$ 0.375 , where the patch numbers corresponding to patch momentum $\vec{k}_{1}$ are given on the ordinate and $\vec{k}_{2}$ on the abscissa. The remaining free momentum $\vec{k}_{3}$ is fixed to the first patch, cf. Fig.6. The divergent momentum structure corresponds to a $p$-wave instability. In the right panel we display the normalized amplitude of the $p$-wave eigenvectors $\left(p_{x}(\vec{k})-\sqrt{3} p_{y}(\vec{k})\right)$ of the triplet- $x$ pair-scattering amplitude along the Fermi surface. The patch number is enumerated on the abscissa.

absolute value) in e.g. the singlet channel becomes

$$
H_{\mathrm{SC}}^{(\mathrm{s})} \propto-\lambda \sum_{\vec{k}, \vec{k}^{\prime}}\left[v_{\vec{k}, \lambda}^{*} f_{\vec{k}}^{\dagger} \Gamma_{0}^{\dagger} f_{-\vec{k}}^{\dagger}\right]\left[v_{\vec{k}^{\prime}, \lambda} f_{\vec{k}^{\prime}} \Gamma_{0} f_{-\vec{k}^{\prime}}\right]
$$

where sublattice/band and spin labels were again suppressed for clarity. The Hamiltonian $H_{\mathrm{SC}}^{(\mathrm{s})}$ can be decoupled by a Hubbard-Stratonovich transformation with a singlet order-parameter field $\psi_{\vec{k}} \sim v_{\vec{k}, \lambda}\left\langle f_{\vec{k}} \Gamma_{0} f_{-\vec{k}}\right\rangle$. Analogous definitions hold for the triplet case, where the decoupling is performed with the vector order-parameter $\vec{d}_{\vec{k}}$, cf. Appendix A 1 . The order parameter symmetry, i.e., the momentum-space Cooper pair structure, is obtained by projecting the eigenvectors $v_{\vec{k}, \lambda}$ onto suitably defined form factors. These can be obtained from the irreducible representations of the point-group of the hexagonal lattice in real space ${ }^{57 / 65}$. From a neighbor-resolved Fourier transform one can obtain momentum-space representations of form factors with well-defined parity. See Appendix A 2 for details. The phase diagram as obtained from an analysis of the leading instabilities is shown in Fig. 77. The overall structure of the phase boundary between intraband $p$-SC and singlet pairing phases agrees nicely with the findings in Ref. 33. While Ref. 33 reports a singlet $s$-wave regime that extends to low doping, the singlet $s$-wave instability appears only for $\delta>0.4$ in our fRG calculations. This finding agrees with the phase diagram obtained in Ref. 67, where the triplet channel was neglected. We interpret this in favor of our present results. A projection of the initial condition onto pairscattering amplitudes in the singlet regime reveals a $d$ wave dominance for $\delta<0.4$ and a subleading $s$-wave, while for $\delta>0.4$ the situation is reversed and the $s$ wave form factor is dominating. The form factor with the largest weight is subsequently enhanced by the flow. We also performed flows at finite temperature, which, however, showed that temperature does not exert an influence on the respective $d$ - or $s$-wave dominance in our flows. Rather, above the critical temperature the sin- 
gular flow is smoothed, which signals the stability of a Fermi liquid ground-state.

Due to lattice symmetry, the intraband $d$-wave solution is doubly degenerate, i.e., the largest eigenvalue of the singlet pair-scattering comes with a two dimensional eigenspace. Projecting onto the form factors given in Appendix A 2, we find that each eigenvector has overlap with the two even-parity nearest-neighbor $d$-wave form factors $d_{x y}(\vec{k})$ and $d_{x^{2}-y^{2}}(\vec{k})$ as defined by Eq. A6 and Eq. A5 . See Fig. 8 for the momentum-space structure of the divergent singlet vertex function and the corresponding eigenvectors of the pair-scattering amplitudes. We note that due to the lack of particle-hole fluctuations in this reduced flow, no longer-ranged intrasublattice pairing correlations develop.

The interband pair-scattering shows odd-parity $p$-wave correlations in the singlet regime, also with degenerate $p$-wave form factors $p_{x}(\vec{k})$ and $p_{y}(\vec{k})$ on nearest-neighbor bonds as defined by Eq. (A7) and Eq. (A8). The interband correlations are in fact substantial close to $\Lambda_{c}$ and comparable in magnitude to intraband correlations. On the level of our fRG-flows this remains unchanged when turning on finite temperature.

At large doping $\delta>0.4$, the leading intraband correlations change from $d$ - to $s$-wave with even-parity nearestneighbor form factor, see Eq. A4 corresponding to an extended $s$-wave pairing instability.

The degeneracies in the case of singlet instabilities are related to lattice symmetries $57 / 66 \mid 67$. Which linear combination is finally realized in the superconducting state needs to be inferred from, e.g., a comparison of groundstate energies ${ }^{68}$ as obtained from mean-field theory. Only when self-energy feedback or counter-terms 6169170 are included in the fRG-flow, symmetry breaking can be accounted for.

The triplet instability for $J_{\mathrm{H}} \lesssim\left|J_{\mathrm{K}}\right| / 2$ is manifested by diverging triplet vertex functions. As noted in Sect. IV] the discrete symmetry of the Kitaev interaction relates the triplet functions among each other. Since the flow stays in the symmetric regime, the triplet vertex functions diverge simultaneously. Moreover, symmetry ensures that the eigenvalues obtained from diagonalizing the triplet pair-scattering are also degenerate. From the current fRG scheme we can thus infer three degenerate $\vec{d}$-vectors, each one corresponding to one of the degenerate triplet channels. As in the singlet case, the true ground state will pick a particular linear combination, which is, however, inaccessible in the employed scheme. Since the Ward identity (see Sect. A 4 derived from the discrete Kitaev symmetry allows reconstruction of two vertex functions from a given one, we only keep the triplet vertex $V_{x}^{(\mathrm{t})}$ in the flow. The vertices $V_{y}^{(\mathrm{t})}$ and $V_{z}^{(\mathrm{t})}$ are obtained from the final result for $V_{x}^{(\mathrm{t})}$.

The triplet instability is dominated by intraband pairing, which in fact corresponds to the $p$-SC solution found in Ref. 33. From an analysis of the pair-scattering amplitude we obtain with a high numerical accuracy the

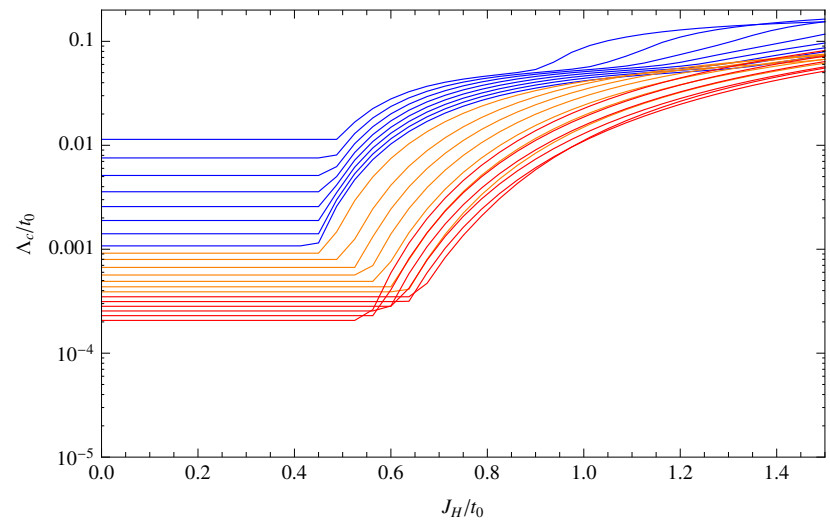

FIG. 10: The critical scale $\Lambda_{0}$ in units of the bare hopping $t_{0}$ as a function of the antiferromagnetic Heisenberg coupling $J_{\mathrm{H}}$, also in units of $t_{0}$ and for fixed $J_{\mathrm{K}} / t_{0}=-1$. The plateaus of constant critical scales below critical Heisenberg coupling can be clearly identified. See main text for an explanation. $\Lambda / t_{0}$ is here given on a logarithmic scale. Starting with doping $\delta=0.1$, the doping level decreases from the top to the bottom curve in steps of $\Delta \delta=0.02$. Blue corresponds to doping $\delta=0.1, \ldots, 0.24$, orange to $\delta=0.26, \ldots, 0.38$, and red to $\delta=0.4, \ldots, 0.5$.

degenerate solutions (see also Fig. 9)

$$
\begin{aligned}
\vec{d}_{\vec{k}, 1} & =\left[p_{x}(\vec{k})-\sqrt{3} p_{y}(\vec{k})\right](1,0,0)^{T}, \\
\vec{d}_{\vec{k}, 2} & =\left[p_{x}(\vec{k})+\sqrt{3} p_{y}(\vec{k})\right](0,1,0)^{T}, \\
\vec{d}_{\vec{k}, 3} & =\left[2 p_{x}(\vec{k})\right](0,0,1)^{T} .
\end{aligned}
$$

Expanding these form factors to leading order in $\vec{k}$ about the $\Gamma$-point in the BZ, we recover the results obtained in Ref. 33 . There it was also shown, that such a $\vec{d}$-vector configuration realizes pairing between fermions with spin projections aligned (with $\sim\left(k_{x}-\mathrm{i} k_{y}\right)$-pairing) or antialigned (with $\sim\left(k_{x}+\mathrm{i} k_{y}\right)$-pairing) along the $(1,1,1)^{T}$ axis in spin space. Further following mean-field arguments 33 and employing knowledge about the mechanism for the creation of topological pairing, namely an odd number of time-reversal invariant points enclosed by the Fermi surface $e^{10 / 11 / 37 / 39}$, for $\delta>0.25$ (indicated by the black dashed line in Fig. 7) the triplet $p$-wave superconductor turns into a topological superconductor.

In total, we obtain good agreement with results from mean-field theory ${ }^{3367}$ from the reduced pure particleparticle flows. Also when turning to the stability of the superconducting phases with respect to thermal excitations, we obtain estimates for critical temperatures from the critical scale $\Lambda_{c}$ (see Fig. 7) that are within the same orders of magnitude as reported in Ref. 33. Within the $p$-wave phase, the critical temperature decreases from $k_{\mathrm{B}} T_{c} \sim 10^{-2} t_{0}$ at $\delta \gtrsim 0.1$ by two orders of magnitude to $k_{\mathrm{B}} T_{c} \sim 10^{-4} t_{0}$ at $\tilde{\delta} \gtrsim 0.3$. For fixed doping level, the critical scale/temperature remains constant within the $p$-wave phase. This, however, can be easily understood 
from the fixed Kitaev coupling $J_{\mathrm{K}} / t_{0}=-1$. Since singlet and triplet vertices are decoupled in the reduced flows, the channel that diverges first wins the race and determines the leading instability. The singlet vertex thus has no influence on the critical scale within the $p$-wave regime determined by the leading triplet channel, see also Fig. 10. For parameters in the singlet regime, the critical scale shows a prominent $J_{\mathrm{H}}$-dependence. Increasing $J_{\mathrm{H}}$, the critical scale/temperature grows rather quickly to larger values $k_{\mathrm{B}} T_{c} \sim 10^{-1} t_{0}$ for $\delta \gtrsim 0.1$. A logarithmic plot of the critical scale as a function of $J_{\mathrm{H}} / t_{0}$ for various dopings is given in Fig. 10, where the plateaus for fixed doping within the triplet regime can be clearly identified.

\section{Unbiased resummation of particle-particle and particle-hole bubbles}

Having established our method in the limit of exclusive particle-particle contributions to the flow of the scaledependent vertex functions, we now include the particlehole fluctuations. These lead to a coupling of singlet and triplet vertex functions. The particle-hole contributions are in fact considerably more complicated than the particle-particle contributions alone. This originates from our choice of channel decomposition of the initial condition, cf. Eq. 15) and Eq. (16).

The resulting phase diagram is presented in Fig. 3. The $p$-wave instability seems to be largely unaffected by the inclusion of particle-hole fluctuations. As in the pure particle-particle case, symmetry guarantees degeneracy of the triplet vertices. We even find that the $\vec{d}$-vector describing the triplet instability is still rather well described by the form given in Eq. (27). Particle-hole fluctuations, however, generate longer-ranged pairing correlations. In the triplet channel, these are subleading contributions compared to the leading nearest-neighbor $p$-wave. For intermediate $J_{\mathrm{H}}$ and $\delta$ the leading instability still occurs in the singlet channel with $d$-wave symmetry, and for larger doping $\delta \gtrsim 0.4$ the order-parameter symmetry switches to $s$-wave. The phase boundaries between the adjacent superconducting instabilities appear to be rather robust with respect to particle-hole fluctuations as compared to the previous pure particle-particle resummation. Critical scales and temperatures are also only mildly affected. We plot the critical scale logarithmically in Fig. 11 for various dopings as a function of $J_{\mathrm{H}} / t_{0}$. We do no longer find a constant $\Lambda_{c}$ for fixed doping and $J_{\mathrm{K}} / t_{0}=-1$ as $J_{\mathrm{H}}$ is varied within the $p$-wave triplet regime. As expected, the particle-hole fluctuations suppress the critical scale in the superconducting regimes. Quantitatively, the changes as compared to the pure particle-particle case reach up to an order of magnitude, cf. Fig. 10. Finally, in the large- $J_{\mathrm{H}}$ regime, the character of the instability changes from superconducting to magnetic. This can be read off from the singlet and triplet vertex functions as shown in Fig. 12 . In the case of spin or charge density wave (SDW, CDW)

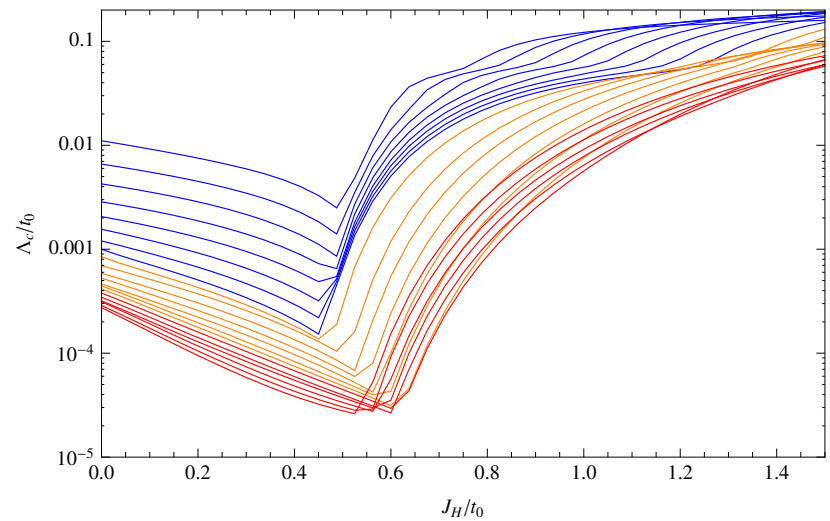

FIG. 11: The critical scale $\Lambda_{0}$ in units of the bare hopping $t_{0}$ as a function of the antiferromagnetic Heisenberg coupling $J_{\mathrm{H}}$, also in units of $t_{0}$ and for fixed $J_{\mathrm{K}} / t_{0}=-1$. $\Lambda / t_{0}$ is here given on a logarithmic scale. Starting with doping $\delta=$ 0.1 , the doping level decreases from the top to the bottom curve in steps of $\Delta \delta=0.02$. Blue corresponds to doping $\delta=0.1, \ldots, 0.24$, orange to $\delta=0.26, \ldots, 0.38$, and red to $\delta=0.4, \ldots, 0.5$.

instabilities, the singlet and triplet vertex functions encode the corresponding divergent momentum structure in a rather complicated way due to the channel decomposition that is adapted to pairing instabilities. Nevertheless, the form of the full vertex function $V^{\Lambda}$ can in these cases be obtained essentially by matrix algebra ${ }^{3}$ and the momentum structures corresponding to SDW and CDW instabilities can be obtained. Using Fierz identities and re-combining singlet and triplet pairing channels, we recover a Hamiltonian

$$
H_{\mathrm{AF}} \propto-V \sum_{o, o^{\prime} \in A, B} \epsilon_{o, o^{\prime}} \vec{S}_{\vec{q}=0}^{o} \cdot \vec{S}_{\vec{q}=0}^{o^{\prime}}, \quad V>0,
$$

that describes the low-energy degrees of freedom close to the critical scale $\Lambda_{c}$. Here, $S_{\vec{q}}^{o}=\sum_{\vec{k}} f_{o, \vec{k}, \sigma}^{\dagger}[\vec{\sigma}]_{\sigma \sigma^{\prime}} f_{o, \vec{k}-\vec{q}, \sigma^{\prime}}$ is the $\vec{q}$-component of the fermion spin operator in sublattice $o \in A, B$. The pre-factor $\epsilon_{o, o^{\prime}}$ equals -1 for $o \neq o^{\prime}$ corresponding to antiferromagnetic correlations between the two sublattices. For $o=o^{\prime}, \epsilon_{o, o^{\prime}}=+1$, which describes ferromagnetic correlations in a given sublattice. The long-range order corresponding to such a Hamiltonian with infinitely ranged interaction is nothing but a two sublattice Néel state, i.e., a commensurate antiferromagnet, where the staggered magnetization is arranged over the two sublattices.

The momentum structure displayed in Fig. 12 is rather broad and smeared out. We confirmed that these features are also obtained from a Hubbard model in the large- $U$

\footnotetext{
3 The actual computations are most efficiently performed with the help of so-called Fierz identities, which can be understood as 're-arrangement' formulas for the index structure of a quartic interaction term.
} 

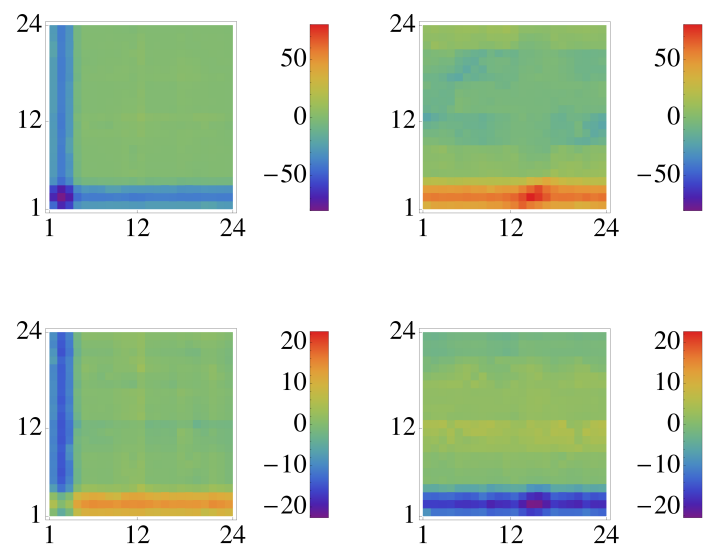

FIG. 12: The upper panel shows the intrasublattice (left) and intersublattice (right) component of the divergent singlet vertex function for $\delta=0.12$ and $J_{\mathrm{H}} / t_{0}=0.75$ with $J_{\mathrm{K}} / t_{0}=-1$, where the patch numbers corresponding to patch momentum $\vec{k}_{1}$ are given on the vertical and $\vec{k}_{2}$ on the horizontal axis. The remaining free momentum $\vec{k}_{3}$ is here fixed to the second patch, cf. Fig. 6 With the same conventions, the lower panel shows the intrasublattice (left) and intersublattice (right) component of the divergent triplet- $x$ vertex function. The divergent momentum structure corresponds to an antiferromagnetic Néel instability. Both amplitude ratio of singlet to triplet vertex and the sign structure conspire to re-combine singlet and triplet pairing interactions into a spin-spin interaction, cf. Eq. (28).

regime on the honeycomb lattice, where the Néel antiferromagnet was established as the magnetically ordered ground-state.

\section{B. Doping QSL and zigzag phase - AF Kitaev and FM Heisenberg exchange}

To analyze the effect of doping charge carriers into the QSL and the magnetically ordered zigzag phase, we select the parameter range of the ferromagnetic $\left(J_{\mathrm{H}}<0\right)$ Heisenberg coupling as $\left|J_{\mathrm{H}}\right| / t_{0} \in[0,1.5]$, while again keeping the now antiferromagnetic Kitaev coupling fixed $J_{\mathrm{K}} / t_{0}=1$. This parameter range covers both QSL and zigzag phase at $\delta=0$. The phase diagram extracted from fRG-flows with both partice-particle and particlehole bubbles is shown in Fig. 4. We again find singlet and triplet pairing instabilities, where as in the case of doping the QSL/stripy phase, the singlet instability comes with different pairing symmetries depending on the doping level. Here, we find two different density-wave regimes (SDW, CDW). A further type on density wave state, a bond-order wave, occurs at the special filling $\delta=1 / 4$, i.e., van Hove filling. This type of instability will be discussed below in Sect. VC after we presented our findings

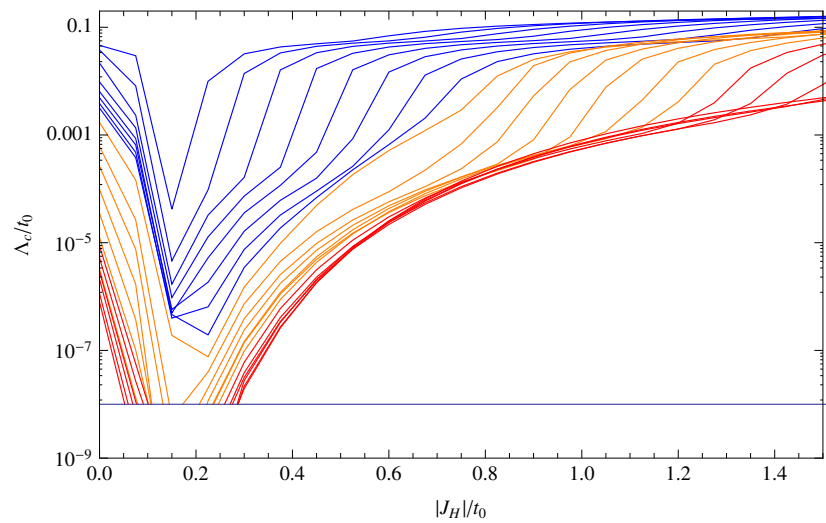

FIG. 13: The critical scale $\Lambda_{0}$ in units of the bare hopping $t_{0}$ as a function of the ferromagnetic Heisenberg coupling strength $\left|J_{\mathrm{H}}\right|$, also in units of $t_{0}$ and for fixed $J_{\mathrm{K}} / t_{0}=1$. $\Lambda / t_{0}$ is here given on a logarithmic scale. Starting with doping $\delta=0.1$, the doping level decreases from the top to the bottom curve in steps of $\Delta \delta=0.02$. Blue corresponds to doping $\delta=0.1, \ldots, 0.24$, orange to $\delta=0.26, \ldots, 0.38$, and red to $\delta=0.4, \ldots, 0.5$. For scales $\Lambda / t_{0}<10^{-8}$, marked by the solid blue line, the fRG-flow could not be evaluated properly to even lower scales for the given choice of parameters used for numerical integration of the flow equation.

for superconducting and SDW/CDW regimes.

At small doping $\delta \simeq 0.1$ and for $\left|J_{\mathrm{H}}\right| / t_{0} \simeq 0.1$, the leading instability is of SDW type. In fact, the divergent momentum structure is the same is in the doped stripy phase, cf. Fig. 12. We thus find a Néel antiferromagnet in this parameter range driven here by the antiferromagnetic Kitaev exchange. As both $\delta$ and $\left|J_{\mathrm{H}}\right|$ are increased, the magnetic order rather quickly makes way for pairing instabilities and an adjacent CDW instability. The vertex structure corresponding to a CDW instability is shown in Fig. 15.

The charge density wave is produced by particle-hole fluctuations, in a similar fashion as the antiferromagnetic instability. A pure particle-particle flow would of course yield a superconducting instability, while a pure particlehole resummation already gives us the CDW instability. The origin of the strong CDW ordering tendencies traces back to the repulsive (for $J_{\mathrm{H}}>0$ ) nearest-neighbor interaction between the sublattice charge densities, see Eq. (4). While we thus find the resulting phase diagram as a 'competition' of tendencies, the existence of either one of the instabilities does not hinge on an interplay between different, competing channels. Such behavior would manifest itself in the complete absence of a particular instability once either particle-particle or particlehole bubbles are excluded from the flow. This observation can be traced back to the $t-J_{\mathrm{K}}-J_{\mathrm{H}}$ model that we take as our starting point. Since important particle-hole fluctuations of a microscopic model in the Mott insulating phase are already contained in the exchange terms, the subsequent fRG-flow tends to enhance the 'pre-formed' tendencies. Similar to the case of the antiferromagnet, 

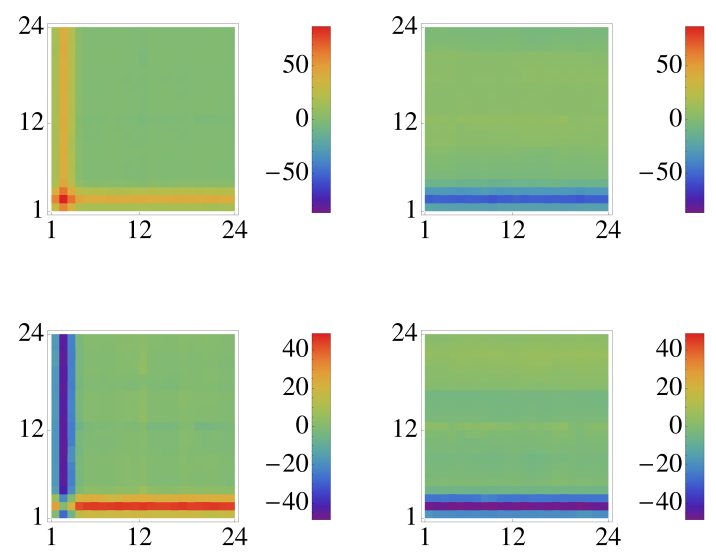

FIG. 14: The upper panel shows the intrasublattice (left) and intersublattice (right) component of the divergent singlet vertex function for $\delta=0.14$ and $\left|J_{\mathrm{H}}\right| / t_{0}=0.525$ with $J_{\mathrm{K}} / t_{0}=1$, where the patch numbers corresponding to patch momentum $\vec{k}_{1}$ are given on the ordinate and $\vec{k}_{2}$ on the abscissa. The remaining free momentum $\vec{k}_{3}$ is here fixed to the second patch, cf. Fig. 6. With the same conventions, the lower panel shows the intrasublattice (left) and intersublattice (right) component of the divergent triplet- $x$ vertex function. The divergent momentum structure corresponds to a CDW instability. Both amplitude ratio of singlet to triplet vertex and the sign structure conspire to re-combine singlet and triplet pairing interactions into a density-density interaction, cf. Eq. 229.

the low-energy degrees of freedom close to $\Lambda_{c}$ can be described by a Hamiltonian of the form

$$
H_{\mathrm{CDW}} \propto-V \sum_{o, o^{\prime} \in A, B} \epsilon_{o, o^{\prime}} N_{\vec{q}=0}^{o} N_{\vec{q}=0}^{o^{\prime}}, \quad V>0 .
$$

Here, $N_{\vec{q}=0}^{o}=\sum_{\vec{k}} f_{o, \vec{k}, \sigma}^{\dagger}\left[\sigma_{0}\right]_{\sigma \sigma^{\prime}} f_{o, \vec{k}-\vec{q}, \sigma^{\prime}}$ is the sublattice density operator for auxiliary fermions. It differs from the electron density only by a factor of $\delta$. The system minimizes its energy by having a charge imbalance, e.g. more electrons reside on sublattice $A$ than on sublattice $B$, or vice versa. The CDW instability takes up a large part of the phase diagram and also comes with rather large critical scales. We estimate critical temperatures up to $k_{B} T_{c} \sim 10^{-1} t_{0}$. Previous mean-field studies did not include CDW order-parameters. In our case, the CDW instability is driven by the density-density term in Eq. (4) for $J_{\mathrm{H}}<0$ and outweighs ferromagnetic ordering tendencies.

We now turn to the superconducting instabilities. The singlet channel determines the leading superconducting instability only in a rather narrow strip for $\left|J_{\mathrm{H}}\right| / t_{0}<$ 0.2 . For doping up to $\delta=0.4$, the intraband pairing symmetry is $d$-wave. Interband correlations are again of $p$-wave type. As the doping level is increased above $\delta=0.4$, an $s$-wave pairing symmetry is favored. Also
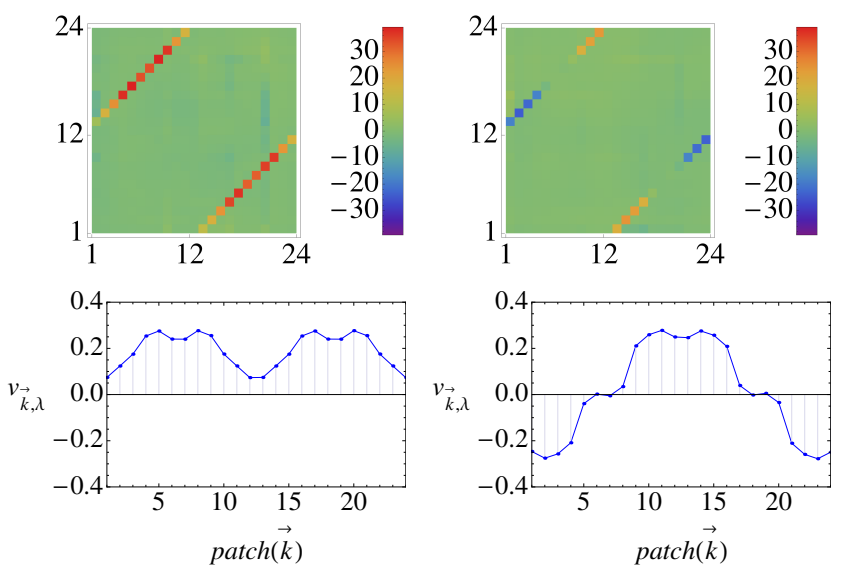

FIG. 15: The upper left panel shows the divergent part of the interband triplet- $x$ vertex function corresponding to $d$ wave pairing symmetry for $\delta=0.34$ and $\left|J_{\mathrm{H}}\right| / t_{0}=0.525$ with $J_{\mathrm{K}} / t_{0}=1$, where the patch numbers corresponding to patch momentum $\vec{k}_{1}$ are given on the ordinate and $\vec{k}_{2}$ on the abscissa. The remaining free momentum $\vec{k}_{3}$ is fixed to the third patch for better visibility, cf. Fig. 6. The upper right panel shows the effect of the proximity to the CDW instability in the intraband triplet- $x$ vertex function. The correlations are of nearest-neighbor $p$-wave type. The lower left panel shows the eigenvector with mixed $s$ - and $d$-wave symmetry of the interband triplet- $x$ pair-scattering amplitude along the Fermi surface. The patch number is enumerated on the abscissa. The lower right panel shows the corresponding intraband eigenvector with $p$-wave symmetry.

here, the dominating superconducting correlations are of nearest-neighbor type. Intrasublattice correlations are subleading.

As the strength of the ferromagnetic Heisenberg coupling is increased, at $\left|J_{\mathrm{H}}\right| / t_{0} \simeq 0.2$ the leading instability switches from singlet to triplet. The different ordering tendencies in particle-hole and particle-particle channels lead to a suppression of intraband-pairing. On moving closer to the triplet-SC/CDW phase boundary, the intraband pairing correlations of $p$-wave type grow stronger (see Fig. 15). Pairing correlations along nearestneighbor bonds still dominate. For the intraband correlations, however, we still observe a substantial decrease as compared to ferromagnetic Kitaev and antiferromagnetic Heisenberg exchange (cf. Sects. V A 1, V A 2), while the interband correlations dominate. The intraband pairing can be described by the following $\vec{d}$-vector

$$
\begin{aligned}
\vec{d}_{\vec{k}, 1} & =\left[p_{x}(\vec{k})+1 / \sqrt{3} p_{y}(\vec{k})\right](1,0,0)^{T}, \\
\vec{d}_{\vec{k}, 2} & =\left[p_{x}(\vec{k})-1 / \sqrt{3} p_{y}(\vec{k})\right](0,1,0)^{T}, \\
\vec{d}_{\vec{k}, 3} & =\left[2 / \sqrt{3} p_{y}(\vec{k})\right](0,0,1)^{T} .
\end{aligned}
$$

As compared to the $\vec{d}$-vector obtained from doping the stripy phase, here the $p$-wave instability is driven by the ferromagnetic and isotropic Heisenberg exchange. 


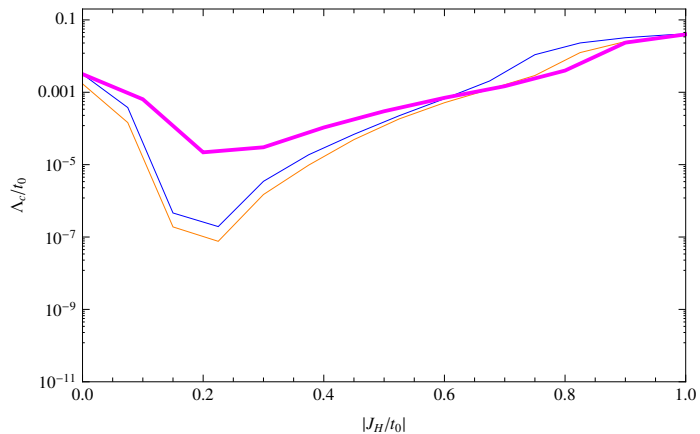

FIG. 16: Critical scale $\Lambda_{c}$ in units of the bare hopping $t_{0}$ for flows evaluated at van Hove filling $\delta=1 / 4$ (magenta) and fillings $\delta=1 / 4-0.01$ (blue) and $\delta=1 / 4+0.01$ (orange) as a function of ferromagnetic Heisenberg exchange $\left|J_{\mathrm{H}}\right| / t_{0}$ and fixed antiferromagnetic Kitaev exchange $J_{\mathrm{K}} / t_{0}=1$. As expected from the enhancement of the single-particle density of states for the nested Fermi surface at van Hove filling, the critical scale is enhanced by a few orders of magnitude. It drops, however, rather quickly as the doping departs from $\delta=1 / 4$. Also, the effect of DOS enhancement reflected in increased critical scales is rendered ineffective as soon as the CDW instability sets in at $\left|J_{\mathrm{H}}\right| / t_{0} \gtrsim 0.7$.

The interband $\vec{d}$-vector is captured by (see also Fig. 15 )

$\vec{d}_{\vec{k}, 1}=\left[-s(\vec{k})+1 / 3 d_{x y}(\vec{k})+1 / 3 \sqrt{3} d_{x^{2}-y^{2}}(\vec{k})\right](1,0,0)^{T}$, $\vec{d}_{\vec{k}, 2}=\left[s(\vec{k})+1 / 3 d_{x y}(\vec{k})-1 / 3 \sqrt{3} d_{x^{2}-y^{2}}(\vec{k})\right](0,1,0)^{T}$, $\vec{d}_{\vec{k}, 3}=\left[s(\vec{k})+2 / 3 \sqrt{3} d_{x^{2}-y^{2}}(\vec{k})\right](0,0,1)^{T}$.

Here, by $s(\vec{k})$ we denote the even parity nearest-neighbor $s$-wave form factor, see Eq. A4). The $p$-wave part in Eq. (30) was reported previously 35 with dominant intraband pairing. We here find dominating interband correlations and enhanced $s$-wave contributions close to the CDW phase boundary.

Critical scales/temperatures for the $p$-wave regime decrease upon doping from $k_{B} T_{c} \sim 10^{-4} t_{0}$ to $k_{B} T_{c}<$ $10^{-8} t_{0}$. Critical scales below $10^{-8} t_{0}$ could actually not be properly resolved from the fRG-flows, see also Fig. 13 . Further, critical scales are not constant along the $J_{\mathrm{H}}$-axis for fixed $\delta$ and $J_{\mathrm{K}}$.

\section{Bond-order instabilities at van Hove filling}

The filling $\delta=1 / 4$ plays a special role in honeycomb lattice models, since perfect Fermi surface nesting and a van Hove singularity coincide. It is thus not surprising, that the effects from nesting and enhanced density of states (DOS) at the Fermi level lead to a strong impact from the particle-hole fluctuations on the emerging Fermi surface instability. Since it is the interplay of nesting and density-of-states enhancement that is important, the ensuing phase at van Hove filling should be considered as rather fragile with respect to deviations in filling factor. It comes, however, with an increased critical scale, i.e., larger critical temperature due to larger Fermi level DOS, cf. Fig, 16. Additionally we find that for the parameter ranges studied in this work, only for antiferromagnetic Kitaev and ferromagnetic Heisenberg exchange do the particle-hole effects outweigh the pairing instability. Consequently, we will focus on $J_{\mathrm{K}}>0$ and $J_{\mathrm{H}}<0$ in the following. As before, we keep the Kitaev interaction fixed at $J_{\mathrm{K}} / t_{0}=1$ and vary the Heisenberg exchange $\left|J_{\mathrm{H}}\right| / t_{0} \in[0,1]$ for fixed doping $\delta=1 / 4$. It turns out that an $N=24$ patching scheme is insufficient to properly capture both DOS enhancement and nesting, and leads to spurious artefact instabilities throughout the phase diagram. Upon increased angular resolution along the Fermi surface, these artefacts disappear at $N=96$ and allow for a clear identification of the resulting ordering structures. Since $N=24$ patching has proven quite reliable in interacting honeycomb systems away from van Hove filling ${ }^{71}$, 73 , we believe our results for $\delta \neq 1 / 4$ are quite robust. We supported this claim by checking the phase boundaries in Fig 4 with $N=96$. Only the singlet/triplet phase boundary was mildly affected.

The nesting vectors $\vec{Q}_{i}, i=1,2,3$ connect opposite edges of the hexagonal Fermi surface at van Hove filling, see Fig. 6. Modulo reciprocal lattice vectors, these are equivalent to vectors connecting inequivalent, neighboring $M$ points. Explicitly, they are given by

$$
\begin{gathered}
\vec{Q}_{1}=\pi(1 / \sqrt{3}, 1)^{T}, \quad \vec{Q}_{2}=\pi(-1 / \sqrt{3}, 1)^{T}, \\
\vec{Q}_{3}=\pi(-2 / \sqrt{3}, 0)^{T} .
\end{gathered}
$$

An emergent order parameter with ordering wavevector $\vec{Q}_{i}$ breaks translation invariance of the underlying lattice and leads to a doubling of the unit cell, i.e., a four atom unit cell in our present case. From analyzing the momentum-space pattern of the renormalized vertex function at the critical scale and employing Fierz identities, we find effective low-energy Hamiltonians of either charge bond-order $(\mathrm{cBO})$ or spin bond-order $(\mathrm{sBO})$ type:

$$
\begin{aligned}
H_{\mathrm{cBO}} & \propto \sum_{i=1}^{3} V_{\mathrm{cBO}}^{i} \Phi_{\vec{Q}_{i}}^{*} \Phi_{\vec{Q}_{i}} \\
H_{\mathrm{sBO}} & \propto \sum_{i=1}^{3} \sum_{l \in\{x, y, z\}} V_{\mathrm{sBO}}^{i, l} \Phi_{l, \vec{Q}_{i}}^{*} \Phi_{l, \vec{Q}_{i}},
\end{aligned}
$$

with charge and spin bond-order amplitudes $V_{\mathrm{cBO}}^{i}$ and $V_{\mathrm{sBO}}^{i, l}$, respectively. The fermionic bilinears $\Phi_{\vec{Q}}$ and $\Phi_{l, \vec{Q}}$ are given by

$$
\Phi_{\vec{Q}} \propto \sum_{\vec{k}, \sigma} \sum_{o, o^{\prime}} \tilde{\epsilon}_{o, o^{\prime}} t_{\vec{k}}^{o, o^{\prime}}(\vec{Q}) f_{o, \vec{k}, \sigma}^{\dagger} f_{o^{\prime}, \vec{k}-\vec{Q}, \sigma}
$$




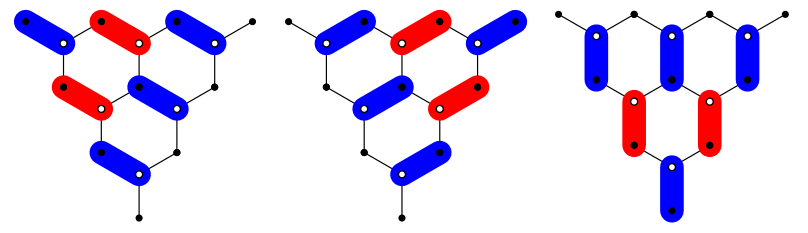

FIG. 17: Real-space charge bond-order patterns for $\left|J_{\mathrm{H}}\right| / t_{0}=$ 0.2 . Red bonds correspond to enhancement of the hopping amplitude along the given bond, while blue bonds correspond to a decrease of the hopping amplitude. White disks mark the $A$-sublattice sites, black disks the $B$-sublattice sites. Shown are the ordering patterns for the three different ordering wavevectors $Q_{1}$ (left), $Q_{2}$ (middle) and $Q_{3}$ (right). The form-factors are given by $\cos \left(\delta_{3} \cdot \vec{k}\right), \cos \left(\vec{\delta}_{1} \cdot \vec{k}\right)$ and $\cos \left(\vec{\delta}_{3} \cdot \vec{k}\right)$, respectively.

and

$$
\Phi_{l, \vec{Q}} \propto \sum_{\vec{k}, \sigma, \sigma^{\prime}} \sum_{o, o^{\prime}} \tilde{\epsilon}_{o, o^{\prime}} t_{l, \vec{k}}^{o, o^{\prime}}(\vec{Q}) f_{o, \vec{k}, \sigma}^{\dagger}\left[\sigma_{l}\right]_{\sigma \sigma^{\prime}} f_{o^{\prime}, \vec{k}-\vec{Q}, \sigma^{\prime}}
$$

where $i=1, \ldots, 3$ labels the different ordering wavevectors, and $l \in\{x, y, z\}$ labels spin-vector components. Here, $\tilde{\epsilon}_{o, \mathrm{o}^{\prime}}=+1$ for $o \neq o^{\prime}$ and $\tilde{\epsilon}_{o, \mathrm{o}^{\prime}}=0$ for $o=o^{\prime}$. This particular form of interlattice correlations corresponds to the dimerization of particle-hole excitations along a given bond. On a mean-field level, a finite expectation value $\left\langle\Phi_{\vec{Q}}\right\rangle$ of the cBO order-parameter leads to a renormalization of the hopping amplitude and to an enlargement of the unit cell with a corresponding downfolded Brillouin zone and additional bands. From numerical calculations, we find the form factors $t_{\vec{k}}^{o, o^{\prime}}(\vec{Q})$ can be described by $\cos \left(\vec{\delta}_{j} \cdot \vec{k}\right), j=1,2,3$ form factors for hopping along nearest-neighbor bonds. The resulting real-space patterns are displayed in Fig. 17. A finite sBO orderparameter $\left\langle\Phi_{l, \vec{Q}}\right\rangle$ leads to a renormalized spin-dependent hopping amplitude. For given $\vec{Q}$, the form factors $t_{\vec{k}}^{o, o^{\prime}}(\vec{Q})$ and $t_{l, \vec{k}}^{o, o^{\prime}}(\vec{Q})$, respectively, determine the bond-order pattern within the enlarged unit cell. From our fRG results we infer the leading instability is either of $\mathrm{cBO}$ or $\mathrm{sBO}$ type, but the two different instabilities do not coincide. The eigenmodes for different $\vec{Q}$ extracted from the corresponding reduced vertex functions - which can again be understood as $N \times N$ matrices - turn out to be degenerate. Further, in the sBO case, there are always two (almost) degenerate eigenmodes with different $l$ for fixed $\vec{Q}$. The association of spin matrices to a given ordering wavevector as obtained from our numerical results is collected in Tab. [1. For sBO instabilities, the dominant features of the numerically obtained form factors can be described with $\sin \left(\vec{\delta}_{j} \cdot \vec{k}\right), j=1,2,3$ where $\vec{\delta}_{j}$ are the nearest-neighbor vectors from $A$ to $B$ sublattice, see Fig. 18. These, of course, can be expressed in terms of nearest-neighbor $p$-wave form factors. The form factors, however, seem to rotate in the degenerate

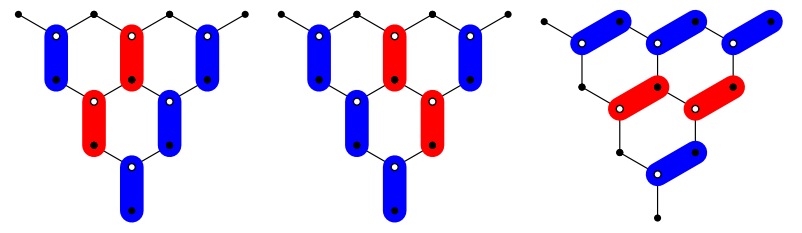

FIG. 18: Real-space spin bond-order patterns for $\left|J_{\mathrm{H}}\right| / t_{0}=$ 0.3. Red bonds correspond to enhancement of the hopping amplitude along the given bond, while blue bonds correspond to a decrease of the hopping amplitude. White disks mark the $A$-sublattice sites, black disks the $B$-sublattice sites. Shown are the ordering patterns for the three different ordering wavevectors $Q_{1}$ (left), $Q_{2}$ (middle) and $Q_{3}$ (right). The spin-dependent form-factors (see Tab. I) are given by $\sigma_{z} \sin \left(\delta_{3} \cdot \vec{k}\right), \sigma_{y} \sin \left(\vec{\delta}_{3} \cdot \vec{k}\right)$ and $\sigma_{x} \sin \left(\vec{\delta}_{1} \cdot \vec{k}\right)$, respectively.

$p$-wave subspace as $J_{\mathrm{H}}$ changes. The modes corresponding to $\cos \left(\vec{\delta}_{j} \cdot \vec{k}\right)$ form factors turn out to be subleading for the sBO instability. Fourier transforming the formfactors yields the corresponding modulation of the realspace hopping amplitude. Due to the limitations of our truncation to the exact hierarchy of fRG equations, we cannot determine which linear combination of the different mean-fields will be realized in the ground state of the system. While some of the sine patterns overlap, others reside on mutually exclusive bonds. For overlapping patterns, we cannot expect the different ordering patterns to be energetically independent. A determination of the lowest-energy configuration, however, is beyond the capabilities of our employed truncation scheme. As displayed in Fig. 4, at small $\left|J_{\mathrm{H}}\right| / t_{0}$ the singlet pairing instability is leading, while at $\left|J_{\mathrm{H}}\right| / t_{0}=0.2$ charge bond-order sets in as the leading instability. As $\left|J_{\mathrm{H}}\right| / t_{0}$ increases, the leading instability quickly crosses over from charge to spin bond-order with the aforementioned two degenerate eigenmodes per ordering wavevector. The bond-order instability is cut off by the CDW for $\left|J_{\mathrm{H}}\right| / t_{0} \gtrsim 0.7$

In view of the superconducting neighborhood of the bond-order instabilities at van Hove filling, cf. Fig. 4, we can infer that while the proximity to even-parity singlet pairing also promotes even-parity singlet charge bondorder, odd-parity triplet pairing favors the formation of odd-parity triplet spin bond-order.

Remarkably, even though we modeled the hopping in the non-interacting Hamiltonian Eq. (2) as spinindependent, the interplay of antiferromagnetic Kitaev and ferromagnetic Heisenberg exchange with nesting and DOS enhancement lead to dynamical re-generation of anisotropic spin-orbit coupling type terms on the level of a mean-field treatment of the low-energy Hamiltonian Eq. (34). While a detailed analysis of the properties of fermions moving in the background of selfconsistently generated bond-order patterns is beyond the scope of this paper, the mean-field Hamiltonian for lowenergy fermions with a static bond-order mean-field readily yields a renormalized fermion spectrum. Considering the different ordering wavevectors independently, we ob- 


\begin{tabular}{c||ccc} 
wavevector & $\sigma_{x}$ & $\sigma_{y}$ & $\sigma_{z}$ \\
\hline$\vec{Q}_{1}$ & $\checkmark$ & - & $\checkmark$ \\
$\vec{Q}_{2}$ & - & $\checkmark$ & $\checkmark$ \\
$\vec{Q}_{3}$ & $\checkmark$ & $\checkmark$ & -
\end{tabular}

TABLE I: Spin matrices associated with the three different ordering wavevectors as obtained from our numerical results. For fixed wavevector $\vec{Q}$, emergent spin bond-order yields spindependent hopping described by the checked spin matrices within a mean-field treatment of the low-energy Hamiltonian Eq. (34.

tain a metallic state for each $\vec{Q}$ with a connected Fermi surface in the reduced Brillouin zone. Energetically, a gapless metallic state might seem less favorable for the system than a state with a nodal superconducting gap. But the condensation of bond order seems to occur at critical scales which are well above the critical temperature for the transition to the superconducting state with a nodal gap along the Fermi surface.

We note that even for $J_{\mathrm{K}}=0$, a finite ferromagnetic Heisenberg coupling $J_{\mathrm{H}}$ is sufficient to drive the system toward a bond-order instability at van Hove filling. A similar observation - ferromagnetic fluctuations causing a propensity toward bond-order instabilities was made for the extended kagome Hubbard model with fRG methods 52 . The dimerization pattern corresponds to spin bond-order, due to the restored rotational symmetry, however, all spin components are degenerate. We attribute the fact that we do not observe a magnetically site-ordered state to the dominating role of the nearestneighbor density-density interaction term, cf. Eq. (4). For $\delta \neq 1 / 4$ and $J_{\mathrm{K}}=0$ critical scales drop quickly below $10^{-8} t_{0}$. The associated instabilities, if they exist, are thus not observable within our current approach.

Finally, we comment on the stability of our results in the presence of Fermi surface renormalization. Since in the present truncation self-energy feedback is completely neglected, the shape of the Fermi surface is fixed during the RG flow. An fRG scheme that takes into account self-energy feedback in principle can modify the Fermi surface and thus might destroy the nesting condition. However, as observed in Ref. 74 the real part of the flowing self-energy mainly leads to a straightening of the Fermi surface. From this point of view, it seems plausible that effects from nesting and DOS enhancement are stable with respect to inclusion of self-energy effects. A more complete picture studying the interplay between van Hove singularities and self-energy flow is, however, certainly desirable.

\section{CONCLUSIONS \& DISCUSSION}

We have analyzed the phase diagram of the doped Kitaev-Heisenberg model on the honeycomb lattice for the situations of ferromagnetic Kitaev and antiferromagnetic Heisenberg exchange, as well as antiferromagnetic
Kitaev and ferromagnetic Heisenberg exchange. We attacked the problem of describing the correlated, frustrated and spin-orbit coupled Mott insulator within a slave-boson treatment, and derived functional RG equations for the auxiliary fermionic problem after the bosonic holon sector was dealt with on a mean-field level. We solved the functional flow equations in the static patching approximation, where the patch number for angular resolution of the Fermi surface ranged from $N=24$ to $N=96$.

While our results corroborate the tendency towards the formation of triplet $p$-wave pairing phases, we demonstrate that other competing orders driven by particle-hole fluctuations reduce the parameter space where pairing yields the leading instability. We further uncovered instabilities at van Hove filling supporting unconventional dimerization phases of the electronic liquid. Interestingly, the prediction of emergent topological $p$-wave pairing states is unaffected by the inclusion of particle-hole fluctuations. For ferromagnetic Kitaev and antiferromagnetic Heisenberg exchange, the gap-closing transition from trivial to topologically non-trivial $p$-wave is left untouched, although critical temperatures are reduced. Flipping the signs of both exchange terms, a bond-order instability pre-empts the naive pairing mean-field gapclosing transition at van Hove filling. The resulting dimerization state, however, remains gapless. Upon doping beyond van Hove filling, the $p$-wave phase is restored. Applying the rule of counting the number of time-reversal invariant momenta below the Fermi surface ${ }^{10 \mid 11 / 37 / 39}$, we again obtain a topological $p$-wave state.

While the dimerized state at van Hove filling appears to remain gapless and non-topological, the proposal of Ref. 32 to include longer-ranged exchange interactions beyond isospins connected by nearest-neighbor bonds to better model the magnetic state of $\mathrm{Na}_{2} \mathrm{IrO}_{3}$ might also provide a route to dynamically generated topological Mott insulating states at van Hove filling.

Extending the $t-J_{\mathrm{K}}-J_{\mathrm{H}}$ model to a $J_{\mathrm{H}}-V$ model in the Heisenberg sector, where $V$ is now promoted to an independent coupling for nearest-neighbor densitydensity interactions (while we restricted our attention to $V=J_{\mathrm{H}}$ ) provides another route to generalization. At least for a subset of initial values for $J_{\mathrm{H}}$ and $V$, however, the fRG-flow will be attracted to the infrared-manifold and the corresponding instabilities we discuss in the present paper. Further, the fRG approach taken in this work might be successfully applied to doping induced instabilities in the context of other material-inspired spinorbit model Hamiltonians 75 .

Before closing the discussion of our results, we briefly comment on the treatment of the $t-J_{\mathrm{K}}-J_{\mathrm{H}}$ model within the fRG framework. The fRG approach employed in this work differs from fRG applications to other, weakly correlated electron lattice systems (for a review, see Ref.42) as its starting point is the renormalized auxiliary fermion Hamiltonian, with a strongly reduced bandwidth. This may cast some doubts on the applicability of a method 
perturbative in the interactions like the fRG. Here, we do not claim that the results are quantitatively controlled, but we can be confident that qualitatively they capture the right trends. First of all it should be noted that using fRG instead of the common mean-field study of the phase diagram of the auxiliary fermion model is certainly an improvement that removes ambiguities and excludes that important channels may get overlooked. Then we also refer to a number of works with a method dubbed 'spin-fRG', cf. Refs. 26 2962. In these works related spin physics is explored in the insulating limit where the kinetic energy is completely quenched. The results obtained there are physically meaningful and give insights into spin physics of frustrated models that are otherwise hard to obtain. In the insulating case, the fermion propagator is purely local and the spin-spin interaction remains of a simpler bilocal form. Hence its full frequency dependence can be taken into account. This simplicity is lost in the doped case studied here, as the fermion propagator is non-local and mediates effective interactions different from simple bilocal spin-spin type. Hence, for us it is difficult to treat the frequency dependence of the vertex in addition to the even more important momentum space structure. Nevertheless, as our case interpolates between the two extreme cases, insulator and weakly correlated systems, where the approach has been shown to work reasonably, we can be confident that studying the correlated doped case by perturbative fRG is justified.

DDS acknowledges discussions with L. Kimme, T. Hyart and M. Horsdal and technical support by M. Treffkorn and H. Nagel. MMS is supported by the grant ERC- AdG-290623.

\section{Appendix A: Technical supplement}

\section{Gamma matrices and superconducting order-parameters}

The decomposition of the interaction terms in the singlet and triplet pairing channels is adapted to analyzing superconducting instabilities. Accordingly, the $\Gamma$ matrices were chosen following the conventions ${ }^{57}$ used in the description of unconventional superconductors. This way, the spin-structure of an emerging superconducting instability is included automatically in our approach. For convenience, we here give the explicit expressions for the complete set of $2 \times 2$ matrices:

$$
\begin{gathered}
\Gamma_{0}=\frac{1}{\sqrt{2}} \sigma_{0} \mathrm{i} \sigma_{y}=\frac{1}{\sqrt{2}}\left(\begin{array}{cc}
0 & 1 \\
-1 & 0
\end{array}\right), \quad \Gamma_{x}=\frac{1}{\sqrt{2}} \sigma_{x} \mathrm{i} \sigma_{y}=\frac{1}{\sqrt{2}}\left(\begin{array}{cc}
-1 & 0 \\
0 & 1
\end{array}\right) \\
\Gamma_{y}=\frac{1}{\sqrt{2}} \sigma_{y} \mathrm{i} \sigma_{y}=\frac{1}{\sqrt{2}}\left(\begin{array}{cc}
\mathrm{i} & 0 \\
0 & \mathrm{i}
\end{array}\right), \quad \Gamma_{z}=\frac{1}{\sqrt{2}} \sigma_{z} \mathrm{i} \sigma_{y}=\frac{1}{\sqrt{2}}\left(\begin{array}{cc}
0 & 1 \\
1 & 0
\end{array}\right) \\
\operatorname{Tr}\left(\Gamma_{\mu} \Gamma_{\nu}^{\dagger}\right)=\delta_{\mu \nu}, \quad \mu, \nu \in\{0, x, y, z\} .
\end{gathered}
$$

Here, $\sigma_{x}, \sigma_{y}$ and $\sigma_{z}$ are the Pauli matrices, and $\sigma_{0}$ denotes the $2 \times 2$ unit matrix. Further, one can derive so-called Fierz identities for this set of matrices in order to re-write quartic pairing terms into density-density type interactions. This enables us to obtain CDW and SDW type instabilities from the singlet and triplet pairing interactions.

The most general superconducting order parameter with singlet and triplet components can now be compactly written as 57

$$
\hat{\Delta}_{\vec{k}}=\sqrt{2}\left(\psi_{\vec{k}} \Gamma_{0}+\vec{d}_{\vec{k}} \cdot \vec{\Gamma}\right)
$$

with $\vec{\Gamma}=\left(\Gamma_{x}, \Gamma_{y}, \Gamma_{z}\right)^{T}$. The singlet order-parameter is described by a scalar function $\psi_{\vec{k}}$, while the triplet orderparameter is specified by a three-component vector $\vec{d}_{\vec{k}}=\left(d_{\vec{k}, x}, d_{\vec{k}, y}, d_{\vec{k}, z}\right)^{T}$. Since in this work we are dealing with a multi-band system, the $2 \times 2$ order-parameter $\hat{\Delta}_{\vec{k}}$ also carries band indices describing intra- $\left(\left[\psi_{\vec{k}}\right]_{b_{1}, b_{2}},\left[\vec{d}_{\vec{k}}\right]_{b_{1}, b_{2}}\right.$ with $\left.b_{1}=b_{2}\right)$ and interband pairing $\left(\left[\psi_{\vec{k}}\right]_{b_{1}, b_{2}},\left[\vec{d}_{\vec{k}}\right]_{b_{1}, b_{2}}\right.$ with $\left.b_{1} \neq b_{2}\right)$.

\section{Form factors on the honeycomb lattice}

The form factors that we employ here for the analysis of order-parameter symmetries are obtained from the irreducible representations of the point group of the hexagonal lattice. For a given representation in terms of functions defined on the real-space lattice, a momentum space form-factor $f_{\vec{k}}$ can be obtained as $f_{\vec{k}}=\sum_{\vec{r}} \mathrm{e}^{\mathrm{i} \vec{k} \cdot \vec{r}} f_{\vec{r}}$. As is usually 
done, the lattice sum is split into nearest-neighbor (NN), next-nearest neighbor (NNN), etc., contributions. The NN form-factors are thus given by $f_{\vec{k}}=\sum_{j} \mathrm{e}^{\mathrm{i} \vec{k} \cdot \vec{\delta}_{j}} f_{\vec{r}+\vec{\delta}_{j}}$. Since the resulting form factors do not come with well-defined parity, we form the appropriate linear combinations yielding form-factors that are either even or odd with respect to $\vec{k} \rightarrow-\vec{k}$. We can proceed accordingly for NNN form factors and so on. The obtained set of form factors is suitable to analyze order-parameter symmetries for fermions in Bloch/sublattice representation. When we switch to the band representation, the NN form-factors pick up a phase factor $\phi_{k}=\sum_{j} \mathrm{e}^{\mathrm{i} \vec{k} \cdot \vec{\delta}_{j}} /\left|\sum_{j} \mathrm{e}^{\mathrm{i} \vec{k} \cdot \vec{\delta}_{j}}\right|$ due to the unitary transformation relating Bloch and band representations. For the NN form-factors in the band representation, we find the following expressions:

$$
\begin{aligned}
s(\vec{k}) & =\frac{1}{3}\left(\cos \left(\frac{\sqrt{3} k_{x}}{2}-\frac{k_{y}}{2}+\phi_{\vec{k}}\right)+\cos \left(\frac{\sqrt{3}}{2} k_{x}+\frac{k_{y}}{2}-\phi_{\vec{k}}\right)+\cos \left(k_{y}+\phi_{\vec{k}}\right)\right) \\
d_{x^{2}-y^{2}}(\vec{k}) & =-\frac{4}{3}\left(\cos \left(\frac{\sqrt{3} k_{x}}{2}-\frac{k_{y}}{2}+\phi_{\vec{k}}\right)+\cos \left(\frac{\sqrt{3}}{2} k_{x}+\frac{k_{y}}{2}-\phi_{\vec{k}}\right)-2 \cos \left(k_{y}+\phi_{\vec{k}}\right)\right) \\
d_{x y}(\vec{k}) & =\frac{8 \sin \left(\frac{\sqrt{3} k_{x}}{2}\right) \sin \left(\frac{k_{y}}{2}-\phi_{\vec{k}}\right)}{\sqrt{3}}, \\
p_{x}(\vec{k}) & =\frac{2 \sin \left(\frac{\sqrt{3} k_{x}}{2}\right) \cos \left(\frac{k_{y}}{2}-\phi_{\vec{k}}\right)}{\sqrt{3}}, \\
p_{y}(\vec{k}) & =\frac{1}{3}\left(-\sin \left(\frac{\sqrt{3} k_{x}}{2}-\frac{k_{y}}{2}+\phi_{\vec{k}}\right)+\sin \left(\frac{\sqrt{3}}{2} k_{x}+\frac{k_{y}}{2}-\phi_{\vec{k}}\right)+2 \sin \left(k_{y}+\phi_{\vec{k}}\right)\right) .
\end{aligned}
$$

In the limit $\phi_{\vec{k}} \rightarrow 0$, we recover the NN form-factors in the Bloch/sublattice representation.

\section{Flow equations}

In this section we summarize the RG-contributions to the right hand sides of Eq. (21) and Eq. (22), respectively. We here stick to the conventions of Ref. 60. We define the shorthand $\int d \tilde{\eta}$ to represent integration/summation over loop variables. The spin projection $\sigma=\uparrow, \downarrow$ is not included and has already been traced over in going from $\xi$ to $\tilde{\xi}$ and $\eta$ to $\tilde{\eta}$. Carrying out the projections onto singlet and triplet channels and defining the loop kernel $L=S^{\Lambda} G_{0}^{\Lambda}+G_{0}^{\Lambda} S^{\Lambda}$ with the single-scale propagator $S^{\Lambda}=d / d \Lambda G_{0}^{\Lambda}$, we find for the singlet case the particle-particle contribution

$$
\phi_{\mathrm{pp}}^{(\mathrm{s})}\left(\tilde{\xi}_{1}, \tilde{\xi}_{2}, \tilde{\xi}_{3}, \tilde{\xi}_{4}\right)=\frac{1}{2} \prod_{\nu=1}^{4} \int d \tilde{\eta}_{\nu} L\left(\tilde{\eta}_{2}, \tilde{\eta}_{1}, \tilde{\eta}_{3}, \tilde{\eta}_{4}\right) V^{(\mathrm{s})}\left(\tilde{\xi}_{2}, \tilde{\xi}_{1}, \tilde{\eta}_{2}, \tilde{\eta}_{3}\right) V^{(\mathrm{s})}\left(\tilde{\eta}_{4}, \tilde{\eta}_{1}, \tilde{\xi}_{3}, \tilde{\xi}_{4}\right)
$$

The particle-particle bubble-contribution to the triplet channel is given by

$$
\phi_{\mathrm{pp} ; l}^{(\mathrm{t})}\left(\tilde{\xi}_{1}, \tilde{\xi}_{2}, \tilde{\xi}_{3}, \tilde{\xi}_{4}\right)=\frac{1}{2} \prod_{\nu=1}^{4} \int d \tilde{\eta}_{\nu} L\left(\tilde{\eta}_{2}, \tilde{\eta}_{1}, \tilde{\eta}_{3}, \tilde{\eta}_{4}\right) V_{l}^{(\mathrm{t})}\left(\tilde{\xi}_{2}, \tilde{\xi}_{1}, \tilde{\eta}_{2}, \tilde{\eta}_{3}\right) V_{l}^{(\mathrm{t})}\left(\tilde{\eta}_{4}, \tilde{\eta}_{1}, \tilde{\xi}_{3}, \tilde{\xi}_{4}\right)
$$

Obviously, particle-particle fluctuations do not couple singlet and triplet vertex-funtions. The singlet particle-hole fluctuations read

$$
\begin{aligned}
\phi_{\mathrm{ph}}^{(\mathrm{s})}\left(\tilde{\xi}_{1}, \tilde{\xi}_{2}, \tilde{\xi}_{3}, \tilde{\xi}_{4}\right)= & -\frac{1}{4} \prod_{\nu=1}^{4} \int d \tilde{\eta}_{\nu} L\left(\tilde{\eta}_{1}, \tilde{\eta}_{2} \tilde{\eta}_{3}, \tilde{\eta}_{4}\right) \times \\
& {\left[V^{(\mathrm{s})}\left(\tilde{\eta}_{4}, \tilde{\xi}_{2}, \tilde{\xi}_{3}, \tilde{\eta}_{1}\right) V^{(\mathrm{s})}\left(\tilde{\xi}_{1}, \tilde{\eta}_{2}, \tilde{\eta}_{3}, \tilde{\xi}_{4}\right)+\sum_{i, j} V_{i}^{(\mathrm{t})}\left(\tilde{\eta}_{4}, \tilde{\xi}_{2}, \tilde{\xi}_{3}, \tilde{\eta}_{1}\right) V_{j}^{(\mathrm{t})}\left(\tilde{\xi}_{1}, \tilde{\eta}_{2}, \tilde{\eta}_{3}, \tilde{\xi}_{4}\right)+\right.} \\
& \left.\sum_{i} V^{(\mathrm{s})}\left(\tilde{\eta}_{4}, \tilde{\xi}_{2}, \tilde{\xi}_{3}, \tilde{\eta}_{1}\right) V_{i}^{(\mathrm{t})}\left(\tilde{\xi}_{1}, \tilde{\eta}_{2}, \tilde{\eta}_{3}, \tilde{\xi}_{4}\right)+\sum_{i} V_{i}^{(\mathrm{t})}\left(\tilde{\eta}_{4}, \tilde{\xi}_{2}, \tilde{\xi}_{3}, \tilde{\eta}_{1}\right) V^{(\mathrm{s})}\left(\tilde{\xi}_{1}, \tilde{\eta}_{2}, \tilde{\eta}_{3}, \tilde{\xi}_{4}\right)\right],
\end{aligned}
$$


while the triplet contribution is given by

$$
\begin{aligned}
\phi_{\mathrm{ph} ; l}^{(\mathrm{t})}\left(\tilde{\xi}_{1}, \tilde{\xi}_{2}, \tilde{\xi}_{3}, \tilde{\xi}_{4}\right)= & -\frac{1}{4} \prod_{\nu=1}^{4} \int d \tilde{\eta}_{\nu} L\left(\tilde{\eta}_{1}, \tilde{\eta}_{2}, \tilde{\eta}_{3}, \tilde{\eta}_{4}\right) \times \\
& {\left[V^{(\mathrm{s})}\left(\tilde{\eta}_{4}, \tilde{\xi}_{2}, \tilde{\xi}_{3}, \tilde{\eta}_{1}\right) V^{(\mathrm{s})}\left(\tilde{\xi}_{1}, \tilde{\eta}_{2}, \tilde{\eta}_{3}, \tilde{\xi}_{4}\right)+\sum_{i, j} c_{i j}^{l} V_{i}^{(\mathrm{t})}\left(\tilde{\eta}_{4}, \tilde{\xi}_{2}, \tilde{\xi}_{3}, \tilde{\eta}_{1}\right) V_{j}^{(\mathrm{t})}\left(\tilde{\xi}_{1}, \tilde{\eta}_{2}, \tilde{\eta}_{3}, \tilde{\xi}_{4}\right)\right.} \\
& \left.\sum_{i} c_{i l} V^{(\mathrm{s})}\left(\tilde{\eta}_{4}, \tilde{\xi}_{2}, \tilde{\xi}_{3}, \tilde{\eta}_{1}\right) V_{i}^{(\mathrm{t})}\left(\tilde{\xi}_{1}, \tilde{\eta}_{2}, \tilde{\eta}_{3}, \tilde{\xi}_{4}\right)+\sum_{i} c_{i l} V_{i}^{(\mathrm{t})}\left(\tilde{\eta}_{4}, \tilde{\xi}_{2}, \tilde{\xi}_{3}, \tilde{\eta}_{1}\right) V^{(\mathrm{s})}\left(\tilde{\xi}_{1}, \tilde{\eta}_{2}, \tilde{\eta}_{3}, \tilde{\xi}_{4}\right)\right] .
\end{aligned}
$$

The four coefficient matrices $c_{i l}$ and $c_{i j}^{l}$ that result from performing internal spin summations encode a specific sign structure,

$$
c_{i l}=\left(\begin{array}{lll}
+1 & -1 & -1 \\
-1 & +1 & -1 \\
-1 & -1 & +1
\end{array}\right)_{i l}, \quad c_{i j}^{x}=\left(\begin{array}{lll}
+1 & +1 & +1 \\
+1 & +1 & -1 \\
+1 & -1 & +1
\end{array}\right)_{i j} \quad c_{i j}^{y}=\left(\begin{array}{lll}
+1 & +1 & -1 \\
+1 & +1 & +1 \\
-1 & +1 & +1
\end{array}\right)_{i j}, \quad c_{i j}^{z}=\left(\begin{array}{lll}
+1 & -1 & +1 \\
-1 & +1 & +1 \\
+1 & +1 & +1
\end{array}\right)_{i j}
$$

and $i, l \in\{x, y, z\}$. The crossed and direct particle-hole contributions entering the flow Eq. 21], Eq. 222) are defined as $\phi_{\mathrm{ph}, \mathrm{cr}}^{(\mathrm{s})}\left(\tilde{\xi}_{1}, \tilde{\xi}_{2}, \tilde{\xi}_{3}, \tilde{\xi}_{4}\right) \equiv \phi_{\mathrm{ph}}^{(\mathrm{s})}\left(\tilde{\xi}_{1}, \tilde{\xi}_{2}, \tilde{\xi}_{3}, \tilde{\xi}_{4}\right)$ and $\phi_{\mathrm{ph}, \mathrm{d}}^{(\mathrm{s})}\left(\tilde{\xi}_{1}, \tilde{\xi}_{2}, \tilde{\xi}_{3}, \tilde{\xi}_{4}\right) \equiv \phi_{\mathrm{ph}}^{(\mathrm{s})}\left(\tilde{\xi}_{1}, \tilde{\xi}_{2}, \tilde{\xi}_{4}, \tilde{\xi}_{3}\right)$ for the singlet case. An analogous definition holds for the triplet case.

Further we note that a delta function taking care of global momentum conservation can be factored out from the flow equations, which leaves only three independent momenta. The final flow equations are formulated and implemented in terms of reduced vertex functions with three independent momenta. For the sake of convenience, we denote full and reduced vertex functions with the same symbol.

\section{Vertex reconstruction from Ward identity}

The symmetries of the Hamiltonian Eq. (14) can be efficiently described by embedding the two-dimensional honeycomb lattice into a three-dimensional cubic lattice ${ }^{34}$. Then a rotation around the $\hat{n}=\frac{1}{\sqrt{3}}(1,1,1)^{T}$ axis by $\pm 2 \pi / 3$ corresponds to the $C_{3}$ or $C_{3}^{-1}$ element, repsectively, of the point group acting on a site in the honeycomb lattice. This rotation also preserves the sublattice index, i.e., both $A$ and $B$ sublattices are mapped onto themselves. We note that the coordinate system is adapted to an embedding of the honeycomb lattice in a 3D cubic lattice ${ }^{34}$. Under a rotation by $-2 \pi / 3$ the spin components along the bonds are mapped as $S_{x} \rightarrow S_{y}, S_{y} \rightarrow S_{z}$ and $S_{z} \rightarrow S_{x}$. As expected from the strong spin-orbit coupling scenario realized in the iridates, the Kitaev term is only invariant under simultaneous transformations of spin and lattice (orbital) degrees of freedom. The corresponding transformation on the lattice is a rotation $R_{\hat{n}}(\theta)$ with $\theta=2 \pi / 3$. This operation can also be represented through combinations of reflections or rotations and reflections. The two transformations (spin and lattice rotation) taken together leave the Hamiltonian Eq. (14) invariant. The $S U(2)$-transformation matrix acting on the fermionic degrees of freedom is given by $S_{\hat{n}}(-\theta)=\exp (\mathrm{i} / 2 \theta \hat{n} \cdot \vec{\sigma})$. Point group transformations acting on the real space lattice also induce a representation on the Brillouin zone. BZ momenta accordingly transform as $\vec{k} \rightarrow \vec{k}^{\prime}=R_{\hat{n}}^{T}(\theta) \vec{k}$.

Moving to a functional formulation and replacing operator valued fields $\left(f^{\dagger}, f\right)$ with Grassmann variables $(\bar{f}, f)$, the symmetry of the system is expressed as the invariance of the generating functional. For the generating functional of $1 \mathrm{PI}$ vertices $\Gamma[\bar{f}, f]$, this statement reads as

$$
\Gamma\left[\bar{f}^{\prime}, f^{\prime}\right]=\Gamma[\bar{f}, f]
$$

where the prime denotes transformed fields. We note that since the kinetic part of the Hamiltonian is also invariant under this rotational symmetry, so are both the bare and the regularized bare propagator in band representation. This in turn implies that the identity Eq. A14 is valid also for the scale-dependent generating functional $\Gamma_{\Lambda}$ for $\Lambda>0$. We thus obtain

$$
\Gamma^{\Lambda}\left[\bar{f}^{\prime}, f^{\prime}\right]=\Gamma^{\Lambda}[\bar{f}, f]
$$

For the discrete rotational symmetry discussed above, we arrive at the following transformation rules for the fermion 
fields in momentum space:

$$
\begin{aligned}
& f_{o, \sigma, \vec{k}} \quad \rightarrow \quad f_{o, \sigma^{\prime}, \vec{k}^{\prime}}^{\prime}=\sum_{\sigma, \vec{k}}\left[S_{\hat{n}}(\theta)\right]_{\sigma^{\prime} \sigma} \delta\left(\vec{k}^{\prime}-R_{\hat{n}}^{T}(\theta) \vec{k}\right) f_{o, \sigma, \vec{k}}, \\
& \bar{f}_{o, \sigma, \vec{k}} \quad \rightarrow \quad \bar{f}_{o, \sigma^{\prime}, \vec{k}^{\prime}}^{\prime}=\sum_{\sigma, \vec{k}}\left[S_{\hat{n}}^{-1}(\theta)\right]_{\sigma \sigma^{\prime}} \delta\left(\vec{k}^{\prime}-R_{\hat{n}}^{T}(\theta) \vec{k}\right) \bar{f}_{o, \sigma^{\prime}, \vec{k}^{\prime}}
\end{aligned}
$$

Expanding both sides of Eq. A15 in transformed and original fields and using the explicit representation of the transformation rules, we find the following set of Ward identities for the (scale-dependent) singlet and triplet vertex functions:

$$
\begin{aligned}
V_{o_{1}, o_{2}, o_{3}, o_{4}}^{(\mathrm{s})}\left(\vec{k}_{1}, \vec{k}_{2}, \vec{k}_{3}, \vec{k}_{4}\right) & =V_{o_{1}, o_{2}, o_{3}, o_{4}}^{(\mathrm{s})}\left(\vec{k}_{1}^{\prime}, \vec{k}_{2}^{\prime}, \vec{k}_{3}^{\prime}, \vec{k}_{4}^{\prime}\right), \\
V_{x ; o_{1}, o_{2}, o_{3}, o_{4}}^{(\mathrm{t})}\left(\vec{k}_{1}, \vec{k}_{2}, \vec{k}_{3}, \vec{k}_{4}\right) & =V_{y ; o_{1}, o_{2}, o_{3}, o_{4}}^{(\mathrm{t})}\left(\vec{k}_{1}^{\prime}, \vec{k}_{2}^{\prime}, \vec{k}_{3}^{\prime}, \vec{k}_{4}^{\prime}\right), \\
V_{y ; o_{1}, o_{2}, o_{3}, o_{4}}^{(\mathrm{t})}\left(\vec{k}_{1}, \vec{k}_{2}, \vec{k}_{3}, \vec{k}_{4}\right) & =V_{z ; o_{1}, o_{2}, o_{3}, o_{4}}^{(\mathrm{t})}\left(\vec{k}_{1}^{\prime}, \vec{k}_{2}^{\prime}, \vec{k}_{3}^{\prime}, \vec{k}_{4}^{\prime}\right), \\
V_{z ; o_{1}, o_{2}, o_{3}, o_{4}}^{(\mathrm{t})}\left(\vec{k}_{1}, \vec{k}_{2}, \vec{k}_{3}, \vec{k}_{4}\right) & =V_{x ; o_{1}, o_{2}, o_{3}, o_{4}}^{(\mathrm{t})}\left(\vec{k}_{1}^{\prime}, \vec{k}_{2}^{\prime}, \vec{k}_{3}^{\prime}, \vec{k}_{4}^{\prime}\right),
\end{aligned}
$$

where we made the orbital indices explicit, i.e., $V_{o_{1}, o_{2}, o_{3}, o_{4}}^{(\mathrm{s})}\left(\vec{k}_{1}, \vec{k}_{2}, \vec{k}_{3}, \vec{k}_{4}\right) \quad \equiv \quad V^{(\mathrm{s})}\left(\tilde{\xi}_{1}, \tilde{\xi}_{2}, \tilde{\xi}_{3}, \tilde{\xi}_{4}\right) \quad$ and $V_{l ; o_{1}, o_{2}, o_{3}, o_{4}}^{(\mathrm{t})}\left(\vec{k}_{1}, \vec{k}_{2}, \vec{k}_{3}, \vec{k}_{4}\right) \equiv V_{l}^{(\mathrm{t})}\left(\tilde{\xi}_{1}, \tilde{\xi}_{2}, \tilde{\xi}_{3}, \tilde{\xi}_{4}\right)$ with $\tilde{\xi}=(\omega, o, \vec{k})$ in the static approximation $\omega=0$. These Ward identities give us the important information, that for one given triplet vertex function, the other remaining two vertex functions can be reconstructed. This fact was exploited in designing an efficient numerical implementation of the flow Eq. (21) and Eq. 222. The Ward identity for the singlet vertex function was not directly employed in the numerical implementation. Besides fermionic exchange symmetry, however, it serves as an important consistency check for the numerical solution of the flow equation.

* Electronic address: daniel.scherer@physik.uni-leipzig.de

1 K. v. Klitzing, G. Dorda, M. Pepper, Phys. Rev. Lett. 45, 494 (1980);

2 R. B. Laughlin, Phys. Rev. B 23, 5632 (1981).

3 D. J. Thouless, M. Kohmoto, M. P. Nightingale, and M. den Nijs, Phys. Rev. Lett. 49, 405 (1982).

${ }^{4}$ Q. Niu, D. J. Thouless, and Y.-S. Wu, Phys. Rev. B 31, 3372 (1985).

5 M. Kohmoto, Ann. Phys. (N.Y.) 160, 343 (1985).

${ }^{6}$ B. A. Bernevig and S.-C. Zhang, Phys. Rev. Lett. 96, 106802 (2006).

7 B. A. Bernevig, T. L. Hughes, S.-C. Zhang, Science, 314, 1757 (2006).

8 A. P. Schnyder, S. Ryu, A. Furusaki, and A. W. W. Ludwig, Phys. Rev. B 78, 195125 (2008).

9 M. Z. Hasan, C. L. Kane, Rev. Mod. Phys. 82, 3045 (2010).

10 X.-L. Qi, T. L. Hughes, S. Raghu, and S.-C. Zhang, Phys. Rev. Lett. 102, 187001 (2009).

11 X.-L. Qi, T. L. Hughes, and S.-C. Zhang, Phys. Rev. B 81, 134508 (2010).

12 S. Ryu et al 2010, New J. Phys. 12065010.

13 L. Balents, Nature 464, 199 (2010).

14 X.-G. Wen and Q. Niu, Phys. Rev. B 41, 9377 (1990).

15 X.-G. Wen, Advances in Physics 44, 405 (1995).

16 D. C. Tsui, H. L. Stormer, and A. C. Gossard, Phys. Rev. Lett. 48, 1559 (1982).

17 F. D. M. Haldane, Phys. Rev. Lett. 51, 605 (1983).

18 R. B. Laughlin, Phys. Rev. Lett. 50, 1395 (1983).

19 B. I. Halperin, Phys. Rev. Lett. 52, 1583 (1984).
20 A. Kitaev, Ann. Phys. (N.Y.) 321, 2 (2006).

21 G. Jackeli and G. Khaliullin, Phys. Rev. Lett. 102, 017205 (2009).

22 J. Chaloupka, G. Jackeli, and G. Khaliullin, Phys. Rev. Lett. 105, 027204 (2010).

23 Yogesh Singh and P. Gegenwart, Phys. Rev. B 82, 064412 (2010).

24 X. Liu et al., Phys. Rev. B 83, 220403(R) (2011).

25 S. Bhattacharjee et al., 2012 New J. Phys. 14073015.

26 Yogesh Singh et al., Phys. Rev. Lett. 108, 127203 (2012).

27 J. Chaloupka, G. Jackeli, and G. Khaliullin, Phys. Rev. Lett. 110, 097204 (2013).

28 M. Kargarian, A. Langari, and G. A. Fiete, Phys. Rev. B 86, 205124 (2012).

29 J. Reuther, R. Thomale, and S. Rachel, Phys. Rev. B 86, 155127 (2012).

30 F. Ye et al., Phys. Rev. B 85, 180403(R) (2012).

31 R. Schaffer, S. Bhattacharjee, and Y. B. Kim, Phys. Rev. B 86, 224417 (2012)

${ }^{32}$ K. Foyevtsova, H. O. Jeschke, I. I. Mazin, D. I. Khomskii, and R. Valenti, Phys. Rev. B 88, 035107 (2013).

33 T. Hyart, A. R. Wright, G. Khaliullin, and B. Rosenow, Phys. Rev. B 85, 140510(R) (2012).

34 Y.-Z. You, I. Kimchi, and A. Vishwanath, Phys. Rev. B 86, 085145 (2012).

35 S. Okamoto, Phys. Rev. B 87, 064508 (2013).

36 L. Kimme, T. Hyart, and B. Rosenow, arXiv:1308.2496 [cond-mat.supr-con].

37 M. Sato, Phys. Rev. B 79, 214526 (2009). 
38 M. Sato and S. Fujimoto, Phys. Rev. B 79, 094504 (2009).

39 M. Sato, Phys. Rev. B 81, 220504(R) (2010).

${ }^{40}$ R. Comin et al., Phys. Rev. Lett. 109, 266406 (2012).

41 Y. K. Kim et al., Science 1251151 (2014).

42 W. Metzner, M. Salmhofer, C. Honerkamp, V. Meden, and Kurt Schönhammer, Rev. Mod. Phys. 84, 299 (2012).

43 P. A. Lee, N. Nagaosa, and X.-G. Wen, Rev. Mod. Phys. 78, 17 (2006).

44 C.-Y. Hou, C. Chamon, and C. Mudry, Phys. Rev. Lett. 98, 186809 (2007).

45 A. G. Grushin, E. V. Castro, A. Cortijo, F. de Juan, M. A. H. Vozmediano, and B. Valenzuela, Phys. Rev. B 87, 085136 (2013).

46 T. C. Lang, Z. Y. Meng, A. Muramatsu, S. Wessel, and F. F. Assaad, Phys. Rev. Lett. 111, 066401 (2013).

47 S. Raghu, X.-L. Qi, C. Honerkamp, and S.-C. Zhang, Phys. Rev. Lett. 100, 156401 (2008).

48 J. Wen, A. Rüegg, C.-C. J. Wang, and G. A. Fiete, Phys. Rev. B 82, 075125 (2010).

49 N. A. García-Martínez, A. G. Grushin, T. Neupert, B. Valenzuela, and E. V. Castro, Phys. Rev. B 88, 245123 (2013).

50 M. Daghofer and M. Hohenadler, Phys. Rev. B 89, 035103 (2014).

51 T. Duric, N. Chancellor, I. F. Herbut, arXiv:1401.5680 [cond-mat.str-el].

52 M. L. Kiesel, C. Platt, and R. Thomale, Phys. Rev. Lett. 110, 126405 (2013).

53 S. Sachdev and R. La Placa, Phys. Rev. Lett. 111, 027202 (2013).

54 J. D. Sau, S. Sachdev, arXiv:1311.3298 [cond-mat.suprcon].

${ }^{55}$ A. Allais, J. Bauer, S. Sachdev, arXiv:1402.4807 [condmat.str-el].

56 A. Allais, J. Bauer, S. Sachdev, arXiv:1402.6311 [condmat.str-el].

57 M. Sigrist and K. Ueda, Rev. Mod. Phys. 63, 239 (1991).

58 C. Platt, W. Hanke, R. Thomale, Adv. Phys. 62, 453, (2013).

59 J. W. Negele, H. Orland, Quantum many-particle systems,
Addison-Wesley Publishing Company (1988).

60 M. Salmhofer and C. Honerkamp, Prog. Theor. Phys. (2001) 105 (1): 1-35.

61 A. Eberlein and W. Metzner, arXiv:1308.4845 [condmat.str-el].

62 J. Reuther, P. Wölfle, Phys. Rev. B 81, 144410 (2010); J. Reuther, R. Thomale, Phys. Rev. B 83, 024402 (2011); J. Reuther et al.,Phys. Rev. B 83, 064416 (2011); R. Suttner, C. Platt, J. Reuther, R. Thomale, arXiv:1303.0579 J. Reuther, R. Thomale, arXiv:1309.3262

63 C. Honerkamp, Phys. Rev. Lett. 100, 146404 (2008).

64 H. Zhai, F. Wang, and D.-H. Lee, Phys. Rev. B 80, 064517 (2009).

65 W.-S. Wang, Y.-Y. Xiang, Q.-H. Wang, F. Wang, F. Yang, and D.-H. Lee, Phys. Rev. B 85, 035414 (2012).

66 M. L. Kiesel, C. Platt, W. Hanke, D. A. Abanin, and R. Thomale, Phys. Rev. B 86, 020507(R) (2012).

67 A. M. Black-Schaffer and S. Doniach, Phys. Rev. B 75, 134512 (2007).

${ }^{68}$ F. Wang, H. Zhai, Y. Ran, A. Vishwanath, and D.-H. Lee, Phys. Rev. Lett. 102, 047005 (2009).

69 R. Gersch et al 2008, New J. Phys. 10045003.

70 M. Ossadnik and C. Honerkamp, arXiv:0911.5047 [condmat.str-el].

${ }^{71}$ M. M. Scherer, S. Uebelacker, and C. Honerkamp , Phys. Rev. B 85, 235408 (2012).

72 T. C. Lang, Z. Y. Meng, M. M. Scherer, S. Uebelacker, F. F. Assaad, A. Muramatsu, C. Honerkamp, and S. Wessel, Phys. Rev. Lett. 109, 126402 (2012).

73 M. M. Scherer, S. Uebelacker, D. D. Scherer, and C. Honerkamp, Phys. Rev. B 86, 155415 (2012).

74 C. Honerkamp, M. Salmhofer, N. Furukawa, and T. M. Rice Phys. Rev. B 63, 035109 (2001).

75 Z. Nussinov, J. van den Brink, arXiv:1303.5922 [condmat.str-el].

76 P. Kopietz, L. Bartosch and F. Schütz, "Introduction to the functional renormalization group," (Springer Verlag, Berlin, 2010). 